\title{
mTOR-dependent translation amplifies microglia priming in aging mice
}

\author{
Lily Keane, ${ }^{1,2}$ Ignazio Antignano', Sean-Patrick Riechers', Raphael Zollinger, ${ }^{2}$ Anaelle A. Dumas, ${ }^{2}$ Nina Offermann, ${ }^{1}$ \\ Maria E. Bernis, ${ }^{1}$ Jenny Russ, ${ }^{1}$ Frederike Graelmann, ${ }^{1}$ Patrick Neil McCormick, ${ }^{1}$ Julia Esser, ${ }^{1}$ Dario Tejera, ${ }^{1}$ Ai Nagano, ${ }^{3}$ \\ Jun Wang, ${ }^{3}$ Claude Chelala, ${ }^{3}$ Yvonne Biederbick, ${ }^{1}$ Annett Halle, ${ }^{1}$ Paolo Salomoni, ${ }^{1}$ Michael T. Heneka, ${ }^{1}$ and Melania Capasso ${ }^{1,2}$
}

${ }^{1}$ Cerman Center for Neurodegenerative Diseases (DZNE), Bonn, Germany. ${ }^{2}$ Centre for Tumour Microenvironment and ${ }^{3}$ Centre for Cancer Cenomics and Computational Biology, Barts Cancer Institute, Queen Mary University of London, London, United Kingdom.

\begin{abstract}
Microglia maintain homeostasis in the brain. However, with age, they become primed and respond more strongly to inflammatory stimuli. We show here that microglia from aged mice had upregulated mTOR complex 1 signaling controlling translation, as well as protein levels of inflammatory mediators. Cenetic ablation of mTOR signaling showed a dual yet contrasting effect on microglia priming: it caused an NF-кB-dependent upregulation of priming genes at the mRNA level; however, mice displayed reduced cytokine protein levels, diminished microglia activation, and milder sickness behavior. The effect on translation was dependent on reduced phosphorylation of 4EBP1, resulting in decreased binding of elF4E to elF4C. Similar changes were present in aged human microglia and in damage-associated microglia, indicating that upregulation of mTOR-dependent translation is an essential aspect of microglia priming in aging and neurodegeneration.
\end{abstract}

\section{Introduction}

Microglia are the immune cells of the brain and play an important role in development, homeostasis, and response to injury (1-4). In the healthy CNS, they continuously survey their microenvironment, and their activation is a 2-step process, requiring loss of inhibitory signals such as CD200 and CX3CL1 from neurons and the triggering of receptors by a range of stimuli (5). Activation is accompanied by upregulation of cell surface receptors as well as production of cytokines. An altered activation state called microglia priming is observed under certain conditions (6); it is a sensitization state, in which microglia have a lower threshold for activation, leading to a stronger inflammatory response. A primed phenotype is observed in the aging brain; consequently, microglia produce more inflammatory cytokines such as TNF- $\alpha$, IL1- $\beta$, and IL- 6 in response to a peripheral challenge (7). Furthermore, microglia appear primed in neurodegenerative diseases (8-11). Indeed, the priming of microglia and the resulting hyper-response to peripheral infection is thought to be an important step in the initiation or progression of neuroinflammatory diseases $(9,12)$. The causes of the primed phenotype remain unclear, and little is known about what signaling pathways regulate it.

Different studies have assessed molecular changes in microglia with age, including from different brain regions (13-16). New studies using single-cell transcriptomics have highlighted microglia heterogeneity at the steady state and in the aging brain, together with the higher inflammatory potential of specific microglia

Authorship note: LK and IA are co-first authors.

Conflict of interest: The authors have declared that no conflict of interest exists. Copyright: () 2021, American Society for Clinical Investigation.

Submitted: August 15, 2019; Accepted: October 14, 2020; Published: January 4, 2021

Reference information: J Clin Invest. 2021;131(1):e132727.

https://doi.org/10.1172/JCl132727. clusters (17). However, insight into potential molecular mechanisms regulating microglia priming remains limited.

mTOR is a serine/threonine kinase of the PI3-K-related family, a central node that controls cellular growth and metabolism (18). It is also implicated in immune responses, such as T cell differentiation, B cell activation (19, 20), and macrophage polarization (16). Inhibition of mTOR by rapamycin was shown to increase the inflammatory response of monocytes and macrophages through increased activation of NF- $\mathrm{KB}(21,22)$. However, other studies have pointed to an antiinflammatory effect of mTOR inhibition, especially in aging (23-25). Inhibition of mTOR has many important biological effects: long-term inhibition increases lifespan $(26,27)$, reduces tumor incidence $(27)$, delays neurodegeneration (28-30), and promotes tolerance in transplant patients (20). On the other hand, short-term inhibition increases immune responses in the elderly (31), highlighting a critical role for mTOR in aging that warrants further investigation because the mode and length of inhibition appear to result in different outcomes.

One of the main functions controlled by mTOR complex 1 (mTORC1) is mRNA translation: mTORC1 regulates cap-dependent translation through its downstream targets, eukaryotic translation initiation factor 4E-binding proteins (4EBP1, 4EBP2, 4EBP3, differentially expressed in different tissues) and S6 kinase, which phosphorylates the ribosomal protein $\mathrm{S} 6$. The 4EBPs bind the eukaryotic translation initiation factor $4 \mathrm{E}$ (eIF4E) and prevent its binding to the mRNA cap and components of the eIF $4 \mathrm{~F}$ initiation complex, such as the scaffold protein eIF4G and the RNA helicase eIF4A (32). Phosphorylation of the 4EBPs by mTORC1 leads to eIF4E release and initiation of translation. Although all eukaryotic mRNAs have a cap, they are not all equally sensitive to $\mathrm{IF} 4 \mathrm{E}$, and therefore mTORC1, regulation (33).

In particular, mRNAs involved in proliferation, cell growth, and immune responses are more sensitive to eIF4E activity (34-38), for 
reasons not fully elucidated (39-45). Overall, translation can regulate immune responses $(38,46)$ and CNS functions $(47,48)$; however, how microglia inflammatory responses are regulated translationally remains to be further clarified.

In this study, we set out to characterize the signaling pathways controlling the microglia-aged phenotype. Our RNA-Seq showed control of translation and the mTOR pathway to be dysregulated with age; this was accompanied by increased mTOR downstream signaling and production of inflammatory cytokines upon peripheral LPS challenge, only at the protein level. Genetic inhibition of mTOR led to an NF- $\kappa \mathrm{B}$-dependent increase in inflammatory and priming mRNAs. In contrast, however, mice showed signs of reduced systemic and microglia-specific inflammation: decreased $4 \mathrm{EBP} 1$ phosphorylation prevented the translation initiation factor eIF4E from interacting with eIF4G, leading to diminished translation of inflammatory mediators.

\section{Results}

The microglia aging phenotype already starts by middle age. In order to study microglia age-dependent changes, cells were purified from the brains of C57BL/6J female mice at 6 months (young), 15 months (middle age), and 23 months (old) of age by FACS (Supplemental Figure 1A; supplemental material available online with this article; https://doi.org/10.1172/JCI132727DS1) and subjected to RNA-Seq (full list of rlog norm counts is available in the NCBI's Gene Expression Omnibus [GEO] database: GSE156762). Microglia preparations had high expression of microglia-specific transcripts, with little or no expression of transcripts specific to other brain cells (Supplemental Figure 1B). Principal component analysis (PCA) showed clustering of samples in an age-dependent manner, with microglia isolated from young, middle-aged, and old mice clustering separately from each other (Figure $1 \mathrm{~A}$ ). We found a number of differentially expressed genes (Figure 1, B and C, and Supplemental Table 1): 805 genes were significantly different specifically between old and young mice, 60 genes between old and middle-aged mice, and 572 genes between middle-aged and young mice (Figure 1B). Interestingly, 344 genes were shared between old and young and middle-aged and young comparisons, highlighting similarities between the middle-aged and old groups (Figure 1B). Many gene expression changes behaved in a monotonic fashion, whereby most were upregulated or downregulated by middle age and then further increased or decreased in old mice (Figure 1, C and D).

Next, we examined genes involved in neuroinflammation, such as cytokines, chemokines, and receptors involved in sensing the neuronal environment. A number of inflammatory genes were upregulated in microglia with age, including the proinflammatory cytokines Csf1, Tnf, Il1a, and Il1b; cytokine receptors such as Il1r and Il2rb2 receptor subunit (Figure 1D); and chemokines (Supplemental Figure 1C). Furthermore, genes involved in microglia priming such as Anxa5, Cd52, Apoe, Axl, Lgals3bp, Tlr2, Clec7a (dectin-1), Cd11c, Cst7, and Spp1 (8), were significantly increased in microglia at 15 months of age, suggesting that microglia were already primed by the middle of the lifespan, with the phenotype becoming more defined by 23 months (Figure 1D). We validated gene expression by qPCR (Supplemental Figure 1D) and flow cytometry (Figure $1 \mathrm{~F}$ ), which showed inflammatory receptors and mediators to be upregulated from as early as 11 months of age, further indicating that microglia priming occurred at an earlier time point than originally reported. Our results are consistent with other transcriptome studies of microglia from the aging brain $(13,17,49)$; however, they suggest that the aging phenotype starts even before mice reach the middle of their lifespan.

The MTOR pathway is upregulated in microglia with age. Ingenuity Pathway Analysis (IPA) of differentially expressed genes between young and aged microglia showed the top 3 dysregulated pathways were related to protein synthesis and mTOR: (a) eIF2 signaling, (b) mTOR signaling, and (c) regulation of eIF4 and p70s6K signaling (Figure 2A). A number of genes were shared across the 3 pathways, such as ribosomal subunit genes and components of the eIF3 initiation complex, all upregulated with age (Figure 2B and Supplemental Table 2). Regarding eIF2 signaling, eIF $2 \alpha$ itself was not upregulated and its kinase eIF2ak1 was mildly upregulated (1.4-fold increase); however, downstream transcription factors such as Atf4 and Ddit3 (coding for CHOP protein) were unaltered (Supplemental Figure 2A). Nonetheless, old microglia showed a mild upregulation of the unfolded protein response (Supplemental Figure 2B). This, together with the small upregulation of eIF2ak1 (Supplemental Figure 2A and Supplemental Table 2), suggested aged microglia can more readily inhibit protein synthesis in response to ER stress; however, no stress response was taking place at the steady state. IPA indicated additional pathways were dysregulated to a smaller extent, confirming an increased inflammatory or primed phenotype (Supplemental Figure 2B and Supplemental Table 3).

Although the RNA-Seq data indicated an effect on mTOR regulation of translation (mTOR signaling, regulation of eIF4 and p70S6K signaling), IPA could not predict whether the pathways were upregulated or downregulated ( $z$ score $<2[ \pm]$, Figure $2 A)$. To clarify this point, we assessed the phosphorylation of the 2 known mTORC1 targets that regulate translation, 4EBP1 and the ribosomal protein $\mathrm{S} 6$, the substrate for the direct mTOR target p70S6 kinase. Phospho-flow cytometry showed an increase in the phosphorylation of 4EBP1 at threonine 37/46 (human nomenclature) in microglia with age (Figure $2 \mathrm{C}$ ), accompanied by a milder increase in S6 phosphorylation at serine 240/244, which showed very high levels in young mice already (Figure 2D). In order to understand what could be causing the activation of MTOR in microglia with age, we measured changes in growth factor concentrations in young and aged brains. Of 30 epithelial and immune-related growth factors, only IGF1 was significantly upregulated in the aging brain, accompanied by a smaller upregulation of the IGF1 binding protein 3 that almost reached statistical significance (Supplemental Figure 2C). Given that IGF1 is a major activator of the mTOR signaling pathway, we speculated that this may account for at least part of the mTOR activation observed in microglia with age.

Phosphorylation of $4 E B P 1$ and S6 are upregulated in microglia from aged mice upon LPS challenge and correlate with increased protein production of inflammatory cytokines. In order to understand whether the age-dependent increase in phosphorylated 4EBP1 (p-4EBP1) and p-S6 affected microglial priming and activation, we challenged young ( 3 months) and old (16 months) mice with a sublethal i.p. dose of LPS and assessed the microglia phenotype after 4 hours. LPS treatment caused an increase in Thr37/46 4EBP1 phosphorylation and Ser240/244 S6 phosphorylation in aged microglia (Figure 3A). Given the role of mTORC1 signaling in regulating translation, we 
A

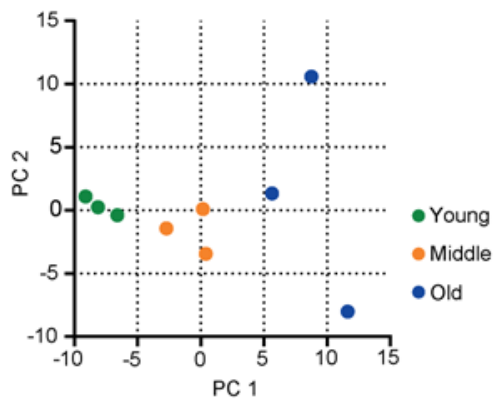

B

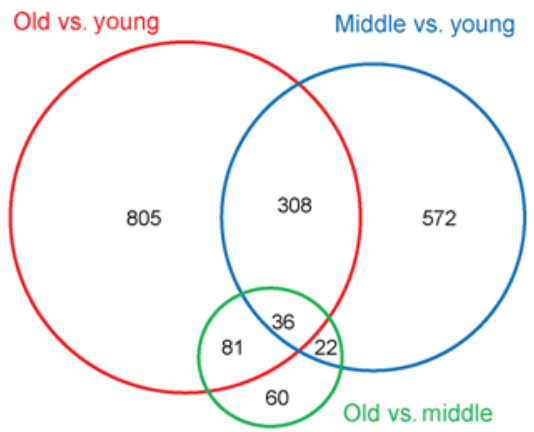

C
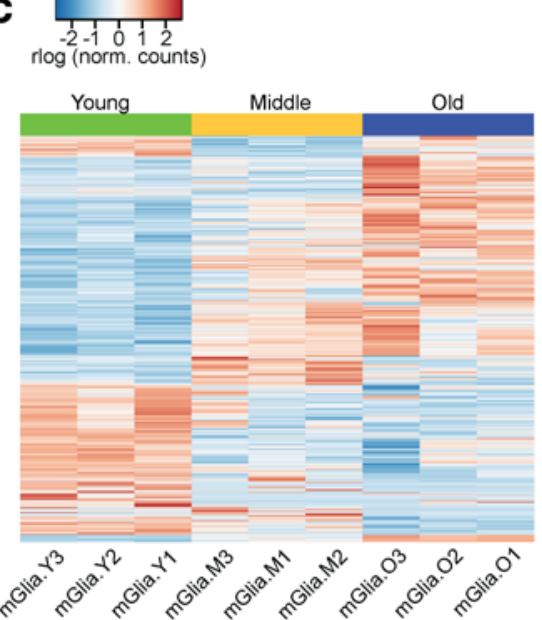

D Cytokines and cytokine receptors
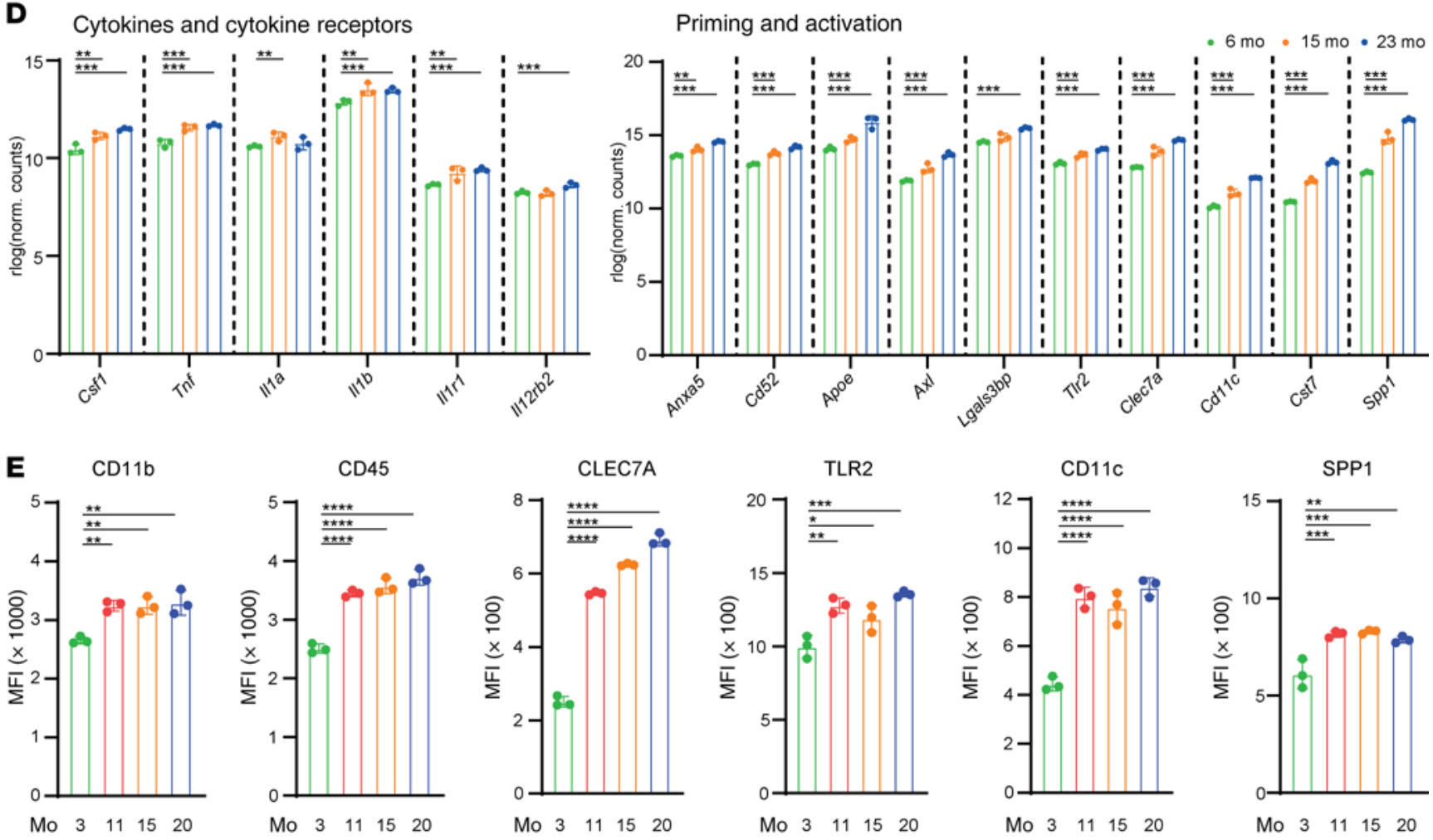

Figure 1. Microglia phenotype changes by middle age. (A) PCA of RNA-Seq of microglia isolated from young (6 months), middle-aged (15 months), and old mice (23 months). (B) Venn diagram showing genes differentially expressed across the age groups, based on DESeq2 FDR-corrected $P$ values. (C) Heat map showing all significant differentially expressed genes identified by DESeq2. (D) Rlog-normalized counts of cytokines, cytokine receptors, and activation and priming genes, based on DESeq2 analysis of RNA-Seq data. (E) Protein validation of genes upregulated in old microglia, measured by flow cytometry and shown as the median fluorescence intensity (MFI). ${ }^{*} P<0.05,{ }^{* *} P<0.01,{ }^{* *} P<0.001,{ }^{* * * *} P<0.0001 ; 1$-way ANOVA ( $n=3$ for all groups).

investigated transcription and translation of inflammatory genes in isolated microglia from young and aged mice after LPS challenge. We found a robust induction of cytokine mRNAs after LPS; however, there were no differences between young and aged microglia at this dose of LPS (Figure 3B, left panels). On the other hand, we found a significant increase in cytokines at the protein level in old microglia (Figure 3B, right panels, and Supplemental Figure 3A). Furthermore, measurement of circulating cytokines showed a similar upregulation in aged mice after LPS injection (Supplemental Figure 3B). In order to exclude that decreased cytokine degradation rather than increased protein output was responsible for the effect, we assessed the autophagy pathway in our RNA-Seq data set and by LC3 staining in microglia from young and aged brains. Our RNASeq data showed no dysregulation of the autophagy pathway with age (Supplemental Figure 3C and Supplemental Table 3). Furthermore, LC3 staining showed a similar number of puncta in microglia from young and aged brains (Supplemental Figure 3D). Taken together, our data suggest that heightened mTOR signaling is present in aged microglia and mediates an increase in their inflammatory response through regulation of translation. 
A

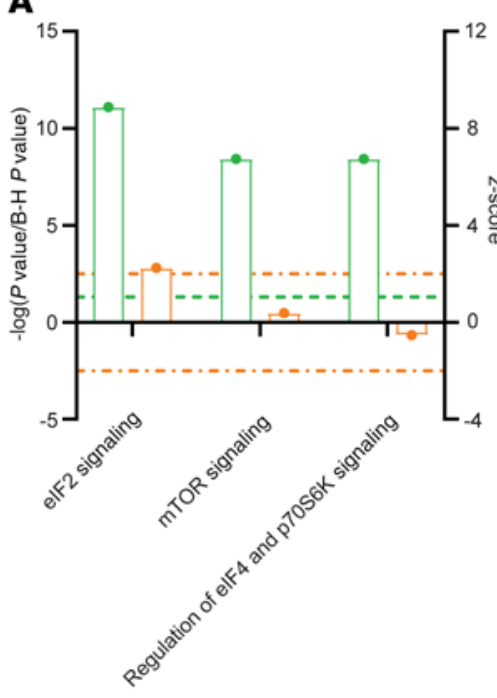

$\square-\log (\mathrm{B}-\mathrm{H} P$ value)

-.- Threshold $-\log (\mathrm{B}-\mathrm{H} P$ value $)$

$\square$ z-score

-.. Threshold z-score
B

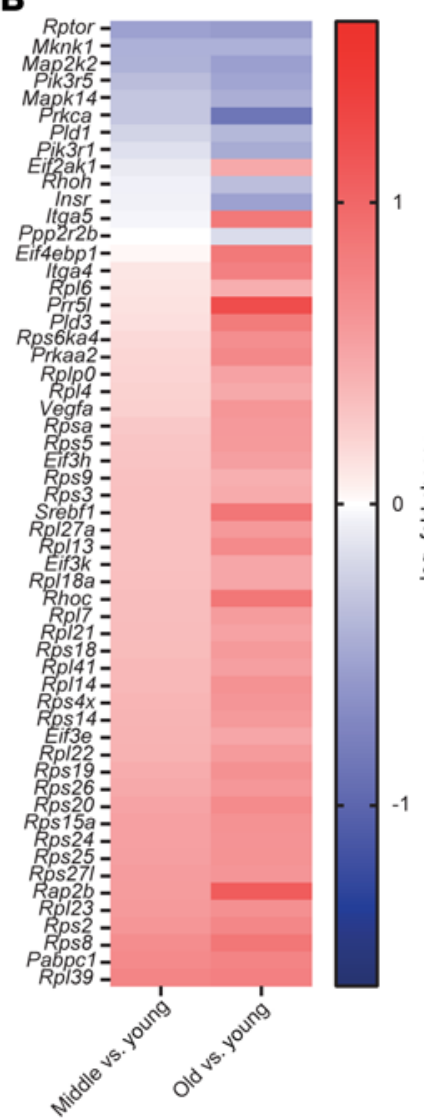

C
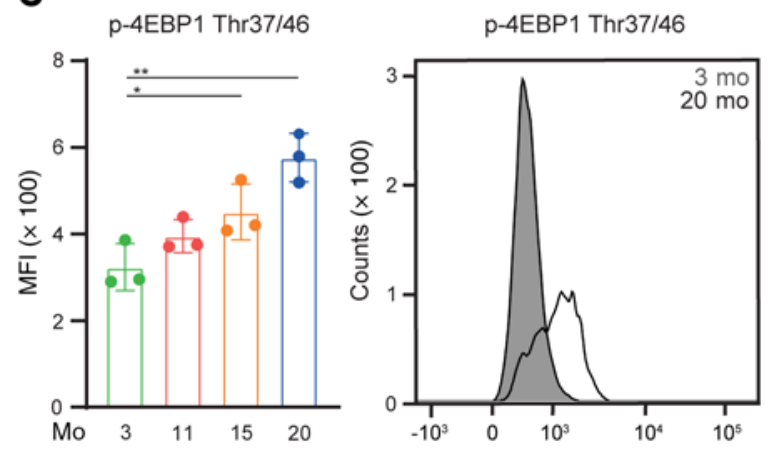

D
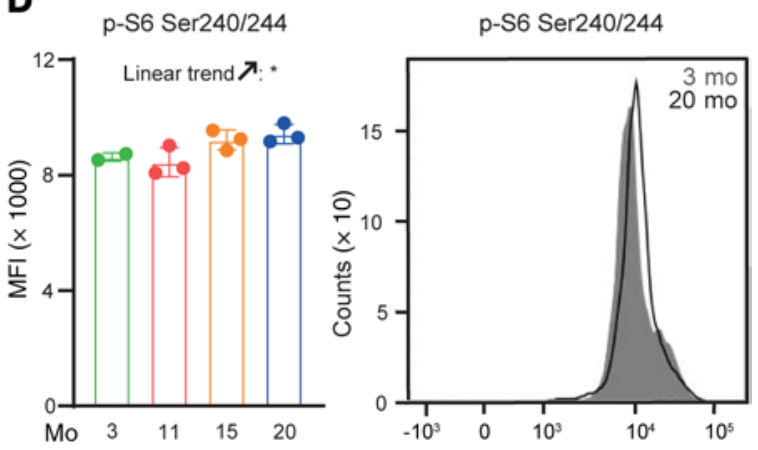

Figure 2. The mTOR pathway is dysregulated in microglia with age. (A) IPA based on all differentially expressed genes between young and old microglia RNA-Seq data, showing the 3 most significantly dysregulated pathways. Data shown in green are $-\log (P$ value $)$ with Benjamini and Hochberg correction for multiple comparisons. For each pathway, the $z$ score is shown in orange. (B) Heatmap showing genes differentially expressed in at least 1 of the 3 most significant IPA pathway hits in middle-aged versus young and old versus young microglia, $n=3$ for both groups. (C and $\mathbf{D})$ Flow cytometry analysis of (C) p-4EBP1 Thr37/46 and (D) p-S6 Ser240/244 in microglia isolated from 3-, 11-, 15-, and 20-month-old female mice shown as MFI. ${ }^{*} P<0.05,{ }^{*} P<0.01$; 1-way ANOVA; $n=3$ for all groups. Panels on the right-hand side are representative histograms for p-4EBP1 and p-S6.

mTORC1 inhibition by genetic deletion of Rheb1 leads to an upregulation of mRNA of inflammatory and priming genes in microglia with aging. In order to assess whether mTORC1 controls the expression of inflammatory and priming genes observed in the aging brain, we used Rheb1/f/l Csfir $r^{\text {Cre }}$ mice (called Rheb-KO for simplicity), in which the GTPase Rheb1, an upstream activator of MTORC1 (50, 51), is deleted in cells expressing Csflr, resulting in RHEB1 loss in the immune system and tissue-resident macrophages, including microglia, as it affects early hemopoietic progenitors (52). Rheb1 deletion was confirmed by recombination of genomic DNA in neonatal microglia (Supplemental Figure 4A), by Western blot in bone marrow-derived macrophages (BMDMs, Supplemental Figure 4B) and neonatal microglia (Supplemental Figure 4C, left and right panel), and by qPCR in cultured neonatal microglia (Supplemental Figure 4D). No effect on Rheb2 (Rhebl1) mRNA expression was observed (Supplemental Figure 4D). Furthermore, Rheb1 loss was confirmed in isolated adult microglia (Supplemental Figure 4E), which also showed reduced p-4EBP1 and p-S6 (Supplemental Figure $4 \mathrm{~F}$ ). Rheb-KO mice were viable, showed no overt phenotype at the steady state, and aged normally (data not shown). They had normal brain and spleen weight (Supplemental Figure 4G) and comparable numbers of all immune cells investigated (Supplemental
Figure $4 \mathrm{H}$ ), including microglia cells in adult and aged brains (Supplemental Figure 4, I and J). This indicated that Rheb1 loss did not affect immune cell proliferation in vivo, although we observed defective proliferation in vitro (Supplemental Figure 4K). Nonetheless, cell size appeared normal, except for a very minor decrease in bone marrow cells (Supplemental Figure 4L).

Previously, it was reported that RHEB1 played a role in mitochondrial clearance through mitophagy (53). In order to assess whether this affected microglia from Rheb-KO mice, we measured mitochondrial mass and intracellular ROS levels. As shown in Supplemental Figure 4M, mitochondrial content and ROS levels were comparable between WT and Rheb-KO microglia from young and old mice. Furthermore, RHEB1 has been implicated in supporting inhibition of protein synthesis upon ER stress by supporting eIF $2 \alpha$ phosphorylation by the kinase PERK (54). In order to assess whether eIF2 $\alpha$ phosphorylation was diminished in Rheb-KO cells during an LPS response, we stimulated BMDMs for the indicated time points (Supplemental Figure $4 \mathrm{~N})$. Steady-state eIF2 $\alpha$ phosphorylation was low in WT and Rheb-KO cells, and it further decreased upon LPS treatment. Nonetheless, no differences could be detected between WT and Rheb-KO BMDMs. These data together confirmed the main defect in Rheb-KO cells is impairment of mTORC1 signaling, as previously shown (51). 
A

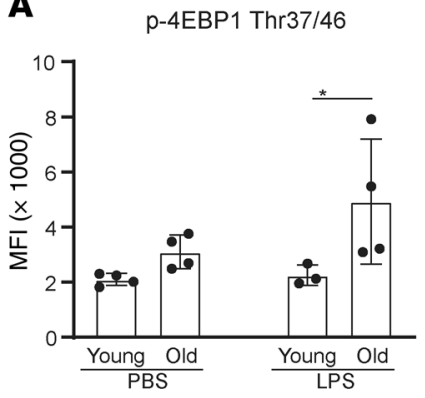

p-S6 Ser240/244

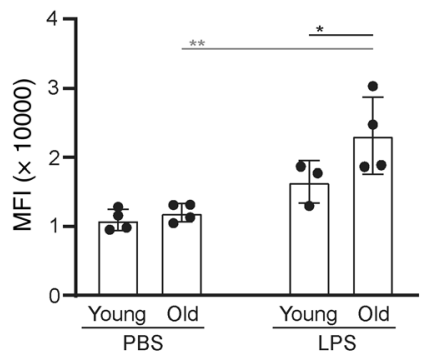

p-4EBP1 Thr37/46

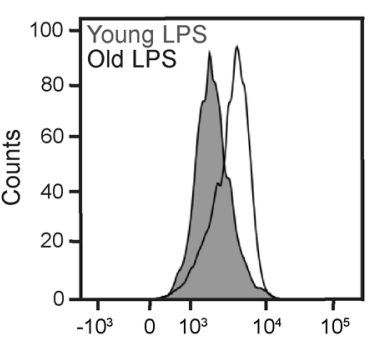

p-S6 Ser240/244

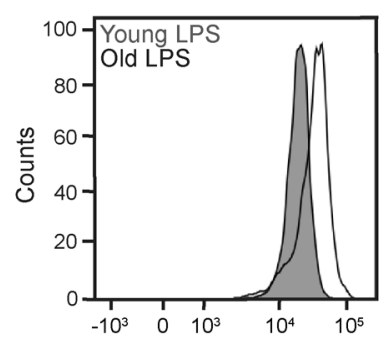

Figure 3. Microglia from LPS-challenged aged mice show increased phosphorylation of 4EBP1 and S6, together with increased cytokine production only at the protein level. Female mice, 3 and 16 months old, were i.p. injected with $5 \mathrm{mg} / \mathrm{kg}$ LPS (or PBS control) and euthanized 4 hours later. (A) Flow cytometry of p-4EBP1 Thr37/46 (upper panels) and p-S6 Ser240/244 (lower panels) in microglia isolated from 3- and 16-month-old mice after LPS or PBS treatment $(n=3-4)$. (B) Gene expression and protein levels of inflammatory cytokines in microglia after in vivo LPS challenge. Left panels: Gene expression measured by qPCR, reported as fold change relative to Hprt expression. Right panels: Protein levels assessed in microglia protein lysates, probed with the Legendplex assay system $(n=4-10)$. ${ }^{*} P<0.05,{ }^{* *} P<0.01,{ }^{* *} P<0.001$, ${ }^{* * *} P<0.0001$; 2 -way ANOVA.

In order to test whether mTORC1 signaling was involved in microglia priming, young and old WT and Rheb-KO mice were i.p. injected with LPS and culled 4 hours later. Quantitative PCR from microglia-derived mRNA showed increased transcription of inflammatory genes, such as Tnf, Il-1b, Il-6, Spp1, Clec7a (dectin-1), and Cst7, after LPS stimulation in vivo in Rheb-KO microglia, further increasing with age (Figure 4A). It has been reported that mTOR inhibition with rapamycin leads to an NF- $\mathrm{NB}$-dependent upregulation of inflammatory genes in human monocytes and mouse macrophages (21). In order to assess whether NF- $\mathrm{kB}$ was also hyperactivated in the absence of RHEB1, we stimulated WT, Rheb-KO, and rapamycin-treated WT BMDMs with LPS and measured nuclear translocation of the NF- $\kappa$ B subunit p65/RelA. Indeed, we found an increase in the translocation of $\mathrm{p} 65$ in Rheb-KO cells comparable to rapamycin treatment, suggesting that $\mathrm{NF}-\mathrm{\kappa B}$ activation might be responsible for the upregulation of cytokine genes in Rheb-KO cells (Figure 4B). Indeed, NF- $\mathrm{kB}$-independent genes, such as Gpnmb and Ccl25, were not upregulated (Figure $4 \mathrm{C}$ ). To further corroborate this finding, we treated macrophages with the NF- $\mathrm{BB}$ peptide inhibitor SN50 and found that it abrogated the increased expression of Tnf in Rheb-KO cells, further indicating that NF- $\kappa \mathrm{B}$ activation was responsible for the increase in cytokine mRNAs in the absence of RHEB1 (Figure 4D).

$m$ TORC1 controls microglia priming by regulating translation of inflammatory mediators. In order to investigate whether cytokine
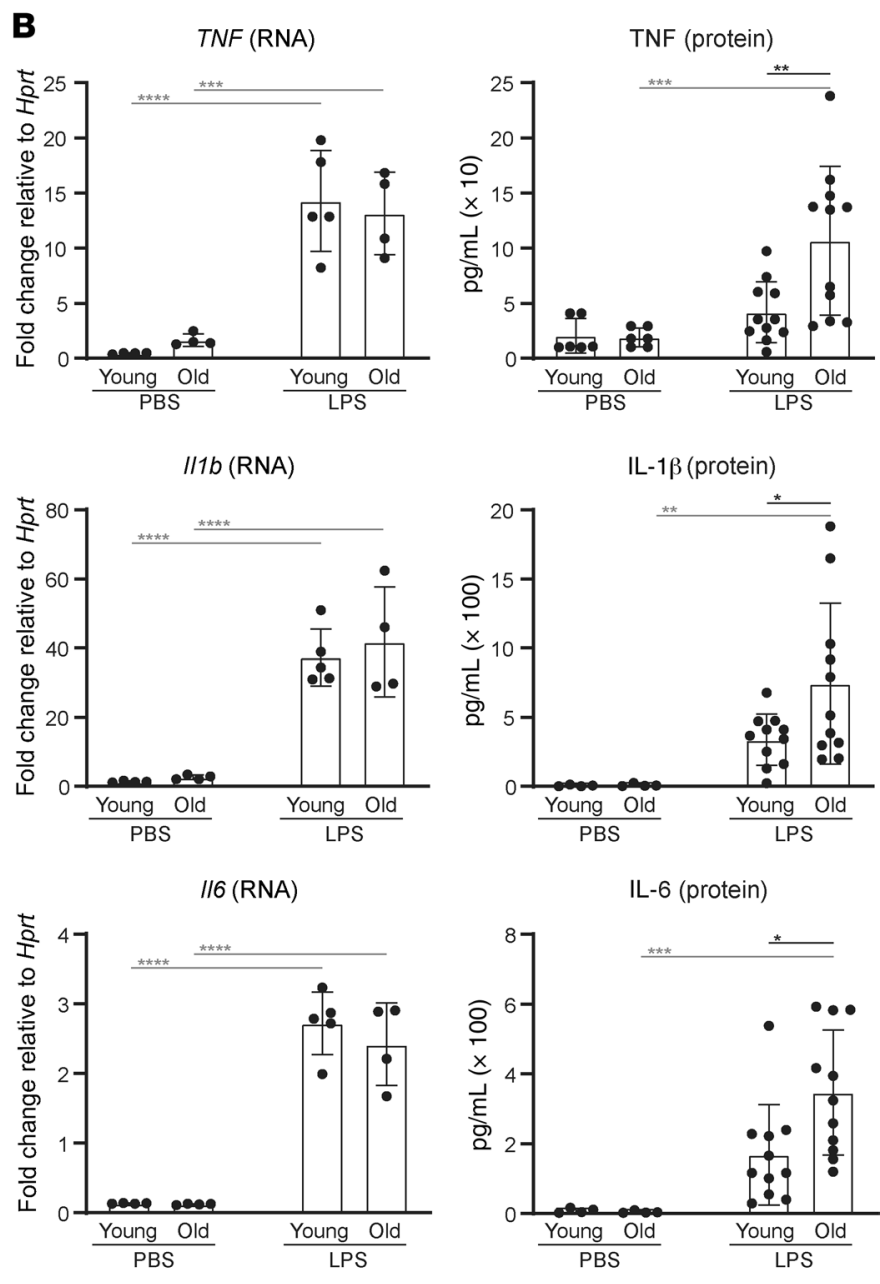

and receptor mRNA upregulation observed in Rheb-KO mice were translated into proteins, we measured protein levels of factors associated with the priming phenotype from WT and Rheb-KO mice upon LPS challenge, such as microglia activation markers (Figure 5A and Supplemental Figure 5A) and systemic inflammatory cytokines (Figure 5B and Supplemental Figure 5B) from young and old mice. In microglia from young mice, we found reduced levels of CLEC7A (DECTIN-1) and SPP1 (Figure 5A), in contrast to their mRNAs (Figure $4 \mathrm{~A}$ ), together with reduced expression of CD11b, CD11c, and TLR2 (Figure 5A). Rheb-KO microglia from aged mice also showed downregulation of inflammatory receptors (Supplemental Figure 5A). Furthermore, plasma levels of inflammatory cytokines, such as TNF and IL12p70 (Figure 5B), IL-1B, IL-1A, and GM-CSF (Supplemental Figure 5B), were diminished in young Rheb-KO mice compared with WT counterparts and even more so in old mice. This further suggested that the transcriptional changes caused by mTORC1 inhibition were not translated into protein. To further clarify whether the net outcome was indeed reduced inflammation in Rheb-KO mice and the potential implications for neurological function, we measured sickness behavior with an open-field test in young mice, monitoring mice behavior and distance traveled while exploring a large empty cage. We found no difference between WT and RhebKO mice at baseline in terms of locomotor activity or anxiety behavior (Supplemental Figure 5C). However, male and female Rheb-KO 

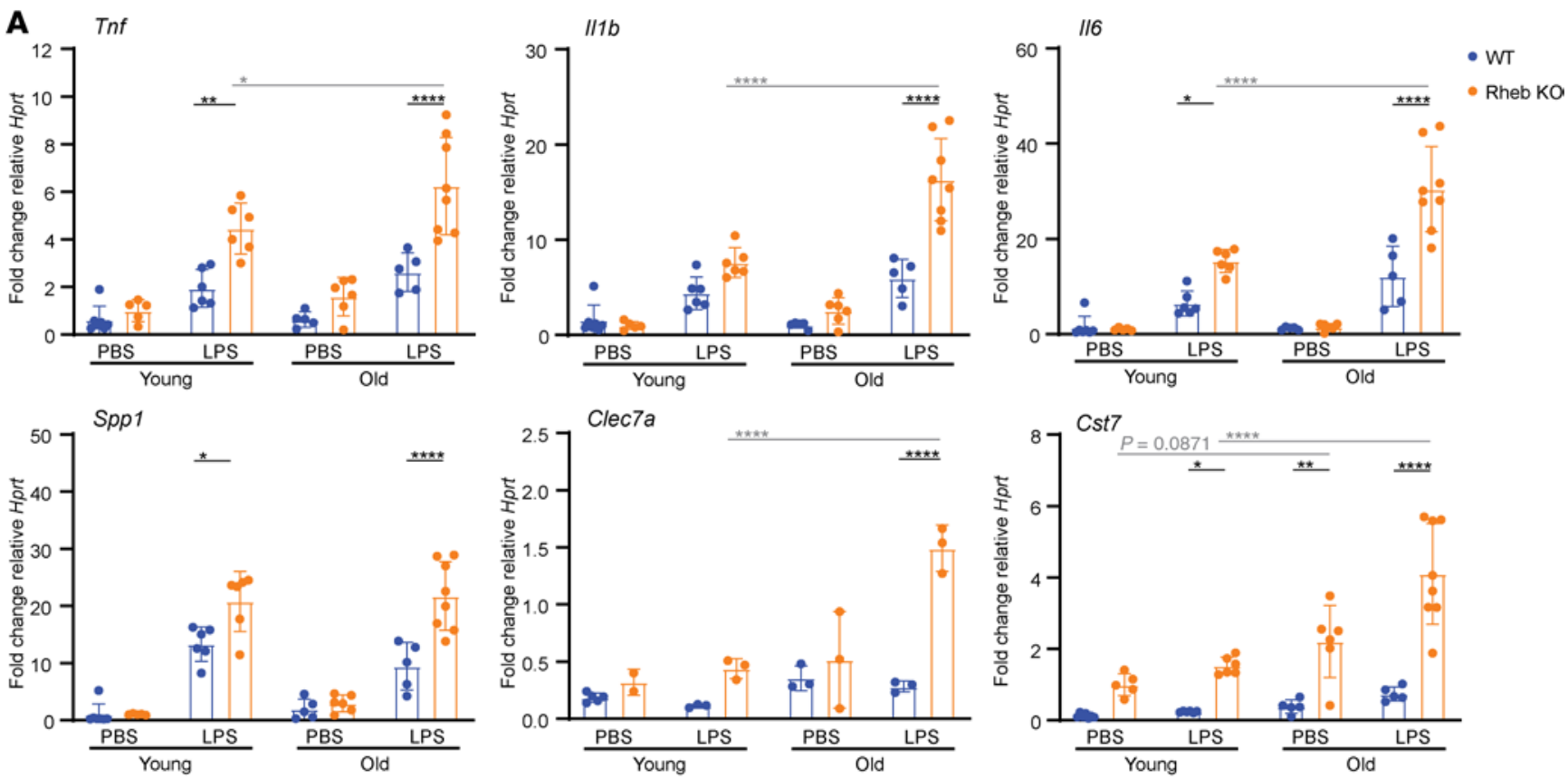

B
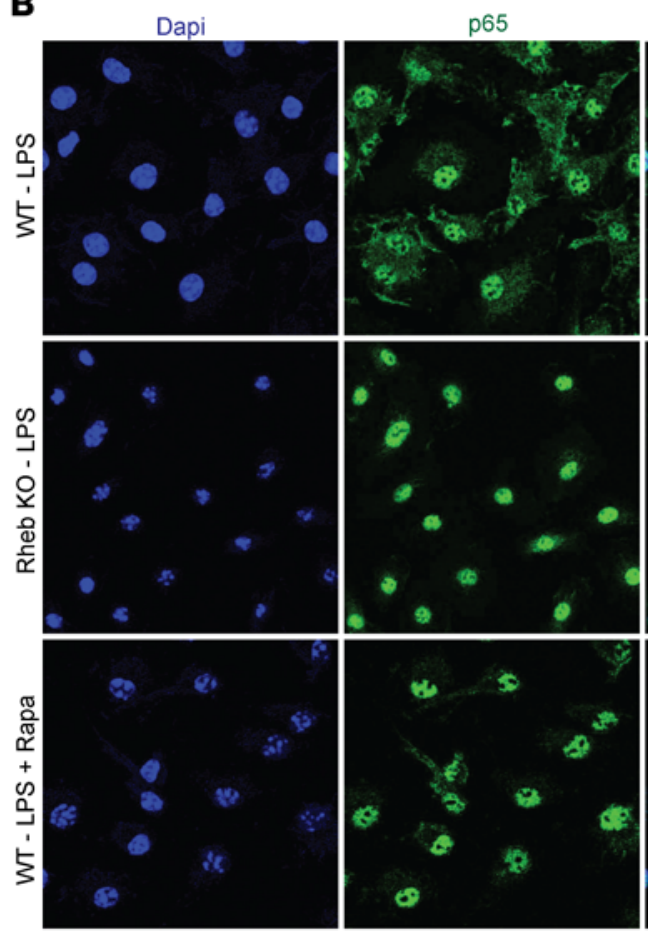

p65

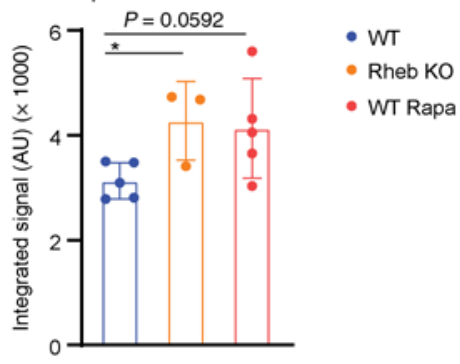

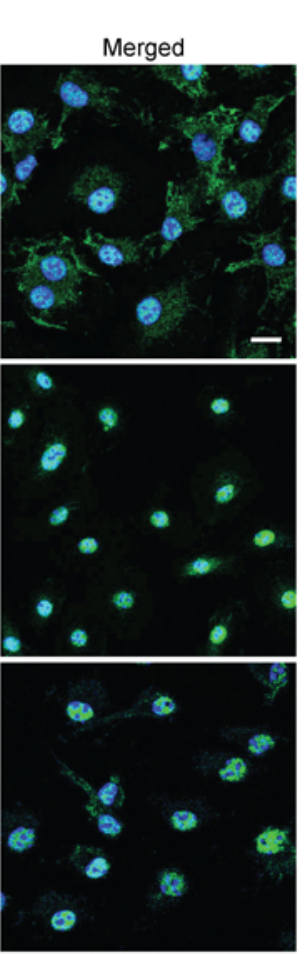

C $G p n m b$
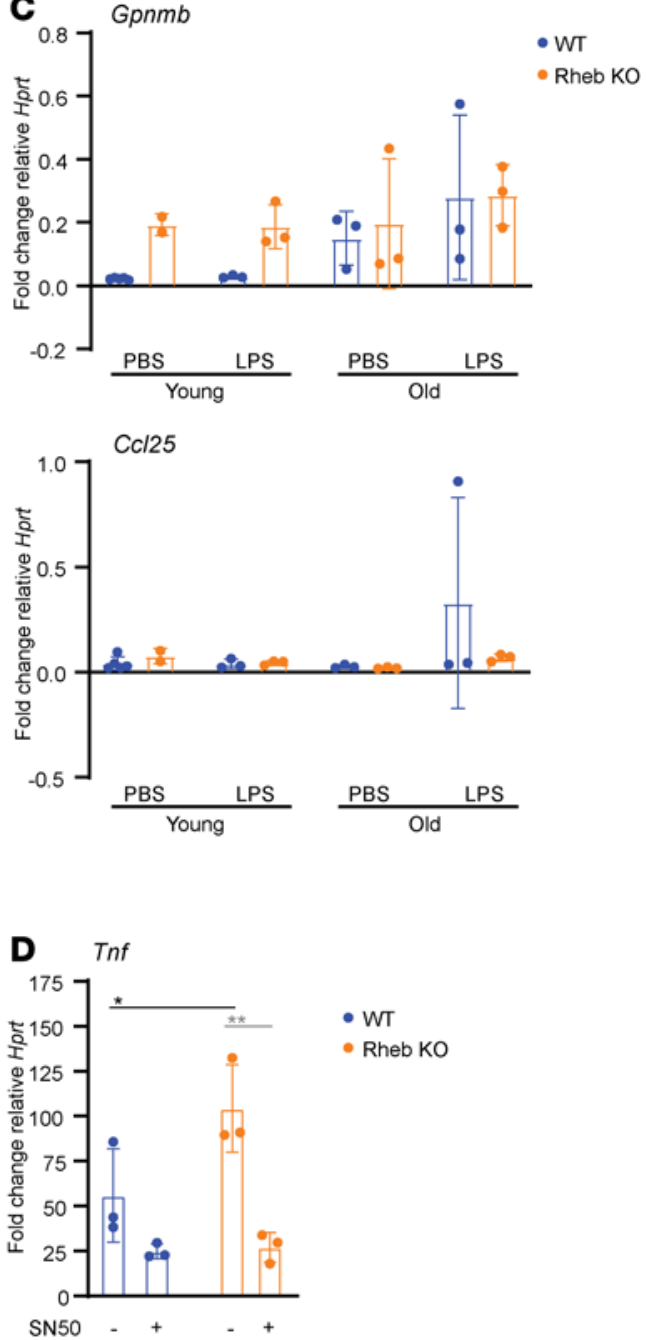
Figure 4. mTORC1 inhibition by genetic deletion of Rheb1 leads to gene expression upregulation of inflammatory and priming genes in microglia. Young (2-6 months old) and aged (15-18 months old) WT and Rheb-KO mice were i.p. injected with $5 \mathrm{mg} / \mathrm{kg}$ LPS or PBS control and euthanized after 4 hours. (A) Expression of inflammatory and age-related genes in microglia assessed by qPCR, reported as fold change relative to Hprt expression. Data shown were collected from 2 independent experiments ( $n$ = 3-8). The results were normalized to young Rheb-KO microglia treated with PBS. (B) Immunofluorescence staining of BMDMs differentiated from WT and Rheb-KO mice for 7 days, then replated and treated with $100 \mathrm{ng} /$ $\mathrm{mL}$ LPS for 6 hours. Nuclei are DAPI stained (blue), NF- $\mathrm{kB}$ p 65 is in green. Scale bar: $10 \mu \mathrm{m}$. Bottom panel: Integrated fluorescence signal of p65 colocalized with DAPI $(n=3-5)$. (C) Expression of NF- $\mathrm{kB}$-independent genes assessed by qPCR in microglia, reported as fold change relative to Hprt expression. Data collected from 2 independent experiments $(n=3)$. (D) Tnf expression as measured by qPCR from BMDMs pretreated with $18 \mu \mathrm{M}$ of the NF- $\mathrm{KB}$ peptide inhibitor, SN50, for 90 minutes and then treated with $100 \mathrm{ng} / \mathrm{mL}$ LPS for 3 hours. Data are shown as the fold change relative to Hprt expression $(n=3)$. ${ }^{*} P<0.05,{ }^{* *} P<0.01$, ${ }^{* * * *} P<0.0001$; 3-way ANOVA (A), Student's $t$ test (B), and 2-way ANOVA (D).

mice displayed milder sickness behavior, illustrated by a significant increase in their locomotor capabilities compared with WT counterparts after LPS injection, such as increased distance traveled and number of rears (Figure 5C). There was no difference in anxiety behavior, measured as time to reach central squares or time spent in them (Supplemental Figure 5D). In order to assess the inflammatory response in Rheb-KO microglia independently of systemic inflammation, we dissected the brain of WT and Rheb-KO mice, in which only microglia cells were $\mathrm{KO}$ for Rheb1, and stimulated acute brain slices with LPS. We found a significant reduction in TNF protein in response to LPS treatment in the absence of RHEB1 in microglia, as measured by ELISA of the culture supernatant (Figure 5D), further confirmed by costaining of the microglia marker IBA1 and TNF by immunofluorescence (Figure 5E). Decreased TNF production was also confirmed in acute brain slices from old mice (Supplemental Figure 5E). Taken together, these results showed that Rheb1 deletion and consequently mTORC1 inactivation led to a reduction in protein levels of inflammatory mediators in microglia cells, despite higher mRNA levels (Figure 4A). The contrasting effect on inflammatory gene transcription and translation caused by Rheb1 loss was also confirmed in BMDMs in vitro (data not shown). In order to assess whether the translational defect was more global and affected cap-dependent translation more generally, we carried out a protein synthesis assay in macrophages using O-propargyl-puromycin in the absence or presence of LPS stimulation. Rheb-KO BMDMs displayed a significant reduction in overall translation 1 hour and 3 hours after LPS treatment compared with WT controls (Figure 5F); however, no significant difference was detected in unstimulated cells, suggesting that mTOR-dependent translation is particularly important in regulating the increased protein output during an inflammatory response. Treatment with cycloheximide, a known inhibitor of translation elongation, completely inhibited protein synthesis in WT and Rheb-KO cells (data not shown), further indicating that loss of Rheb1 resulted in a more selective inhibition of translation upon cell stimulation.

Since the mTOR pathway also controls protein degradation through autophagy, we carried out further experiments to assess whether impairment in protein degradation could account for reduced protein levels of inflammatory mediators in Rheb-KO cells. To this end, WT and Rheb-KO BMDMs were treated with cycloheximide for different time points 90 minutes after LPS stimulation, in order to assess changes in protein levels in the absence of new protein input. As illustrated in Supplemental Figure 6A, the degradation rate did not differ between WT and Rheb-KO cells. In order to further confirm that autophagy was not involved in the observed phenotype, LPS-stimulated WT and Rheb-KO BMDMs were cultured in the presence of bafilomycin A1, an inhibitor of the autophagic flux. No increase in cytokine levels was observed, rather a decrease in both WT and Rheb-KO cells was observed (Supplemental Figure 6B). Interestingly, Rheb-KO cells showed no increase in autophagy compared with WT counterparts (Supplemental Figure 6C), in agreement with a previous report in cardiomyocytes (55). In conclusion, our data indicate that mTORC1 regulates the microglial priming response at multiple levels; however, its control of translation is the critical step in determining the net inflammatory outcome.

Diminished phosphorylation of $4 E B P 1$ causes reduced binding of the translational initiation factor eIF4E to its partner eIF4G in Rheb-KO microglia, leading to impaired translation of cytokines. Our data showed that loss of Rheb1 caused a significant decrease in the translation of inflammatory genes in microglia, on the other hand, rapamycin treatment caused an increase in IL-12p40 (Supplemental Figure 7, A and B), in line with previous publications (21). In order to assess whether this was due to differential regulation of $4 \mathrm{EBP} 1$ phosphorylation, we compared the effect of Rheb1 loss, the ATP-competitive inhibitor Torin1 (56), and rapamycin on the main phosphorylation sites of 4EBP1 (Figure 6 and Supplemental Figure 7, C-G). 4EBP1 Thr37/46 are the first sites to be phosphorylated, followed by Thr70 and Ser65 (57). All phosphorylation sites are believed to be mTOR substrates $(57,58)$, although they show different sensitivity to Torin1 and rapamycin. In particular, Thr37/46 are not sensitive to rapamycin in certain cell types (56-62), whereas Thr70 can be phosphorylated by other kinases, such as ERK2 (63). Western blot and relative densitometry analysis showed a decrease in Thr37/46 in Rheb-KO cells (Figure 6, $\mathrm{A}$ and $\mathrm{B})$, whereas rapamycin did not cause a significant decrease in Thr37/46 (Supplemental Figure 7, C and D). Thr70 phosphorylation appeared mildly downregulated by Rheb1 genetic loss (Figure 6 , A and C), whereas it did not decrease upon rapamycin treatment at any time point investigated (Supplemental Figure 7, C and E). On the other hand, phosphorylation at Ser65 was strongly inhibited in Rheb-KO cells and rapamycin-treated WT cells (Figure 6, $A$ and D, and Supplemental Figure 7, C and F). Inhibition of mTOR with Torin1 reduced Thr37/46 phosphorylation more strongly than Rheb1 loss (Figure 6, F and G). Thr70 phosphorylation was not significantly inhibited by Torin1 (Figure 6, F and H), whereas Ser65 was strongly inhibited, as in the other 2 conditions (Figure 6, F and I). Lastly, changes in phosphorylation levels correlated with an upregulation of 4EBP1 levels in Rheb-KO cells (Figure 6, A and E) and Torin1-treated cells (Figure 6, F and J), whereas no difference in total 4EBP1 was observed in rapamycin-treated cells (Supplemental Figure 7, C and G). In summary, Rheb1 loss and Torin1 treatment caused quantitatively different but qualitatively similar changes in 4EBP1 phosphorylation, accompanied by an increase in 4EBP1 levels. On the other hand, rapamycin did not inhibit 

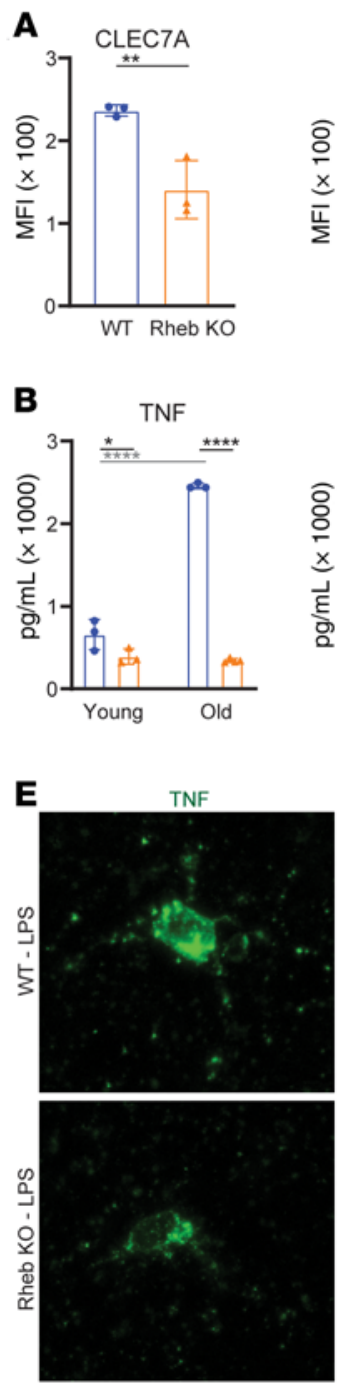

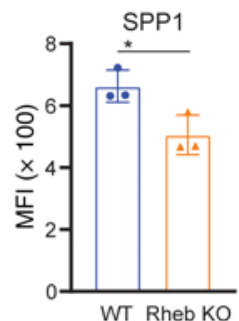

IL12p70

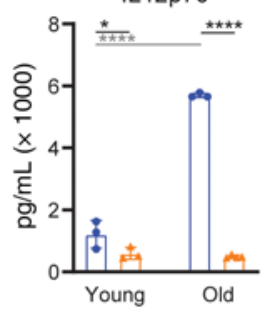

C

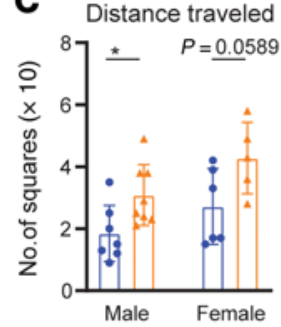

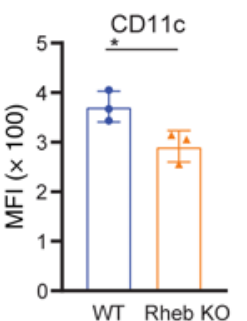
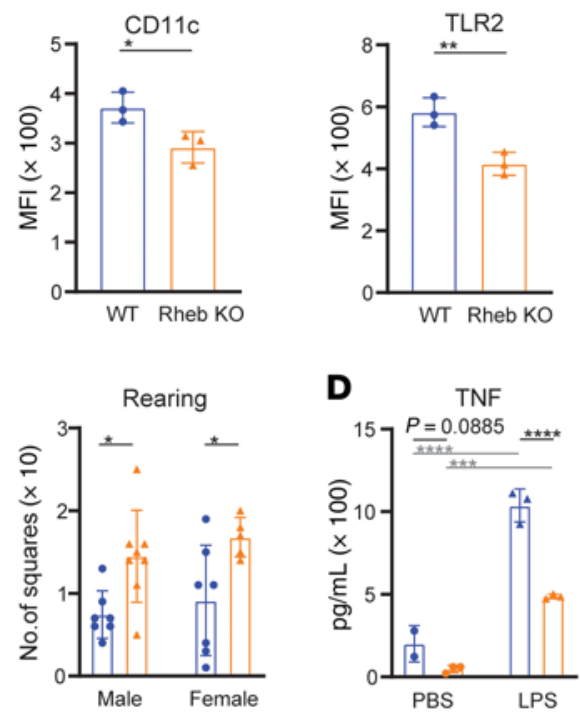
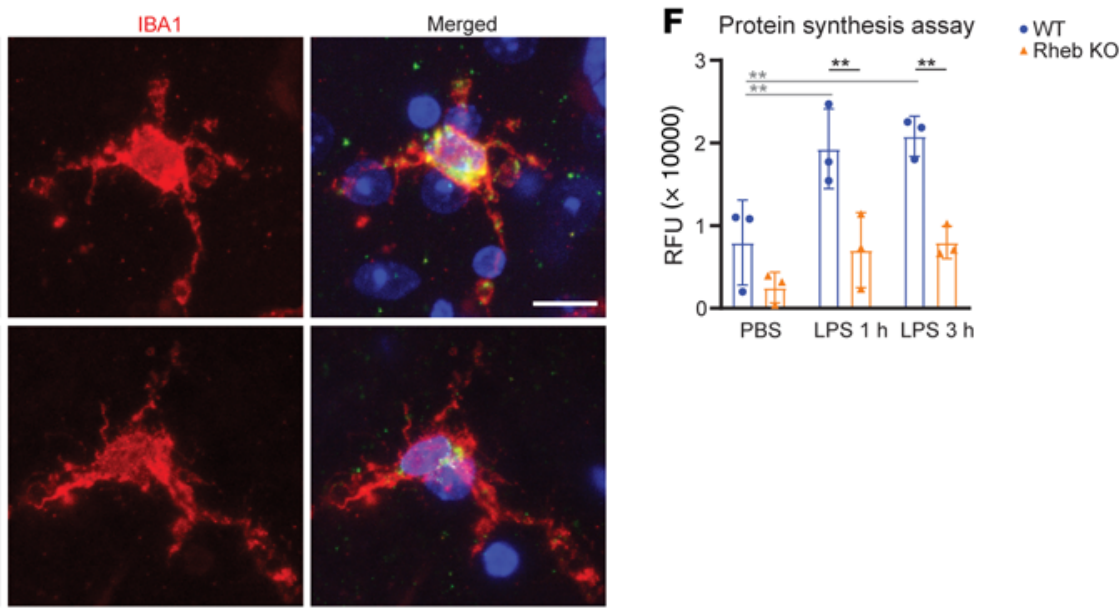

Figure 5. mTORC1 exerts a stronger control over the inflammatory response through translation. (A) Microglia activation markers as assessed by flow cytometry of microglia from WT and Rheb-KO mice. Data are shown as MFI $(n=3)$. (B) Plasma levels of cytokines from young (2-6 months) and aged (15-20 months) WT and Rheb-KO mice, 4 hours after i.p. injection of $5 \mathrm{mg} / \mathrm{kg} \mathrm{LPS}(n=3)$. Cytokines were measured using a Legendplex assay system. (C) Results from open-field test of WT and Rheb-KO mice 6 hours after i.p. injection of $0.33 \mathrm{mg} / \mathrm{mL}$ LPS. Locomotor capabilities were assessed by the distance traveled by each mouse (number of squares it passes through) or by the number of times the mouse reared during the 5-minute test ( $n=5$-8). (D) TNF levels measured by ELISA from culture supernatants of acute brain slices from WT and Rheb-KO mice, after 6 hours of culture with $2 \mu \mathrm{g} / \mathrm{mL}$ LPS ( $n=3$ ). (E) Immunofluorescence staining of acute brain slices from WT and Rheb-KO mice treated with $2 \mu \mathrm{g} / \mathrm{mL}$ LPS for 6 hours. Green: TNF, red: IBA1 (microglia marker). Scale bar: $10 \mu \mathrm{m}$ (objective 40x). (F) Relative fluorescence intensity (RFU) of proteins synthesized in WT and Rheb-KO BMDMs at the steady state and after stimulation with $100 \mathrm{ng} / \mathrm{mL}$ LPS $(n=3) .{ }^{*} P<0.05,{ }^{* *} P<0.01,{ }^{* *} P<0.001,{ }^{* * * *} P<0.0001$; Student's $t$ test $(\mathbf{A}$ and $\mathbf{C})$ and 2-way ANOVA (B, D, and F).

Thr37/46 nor Thr70 phosphorylation and did not cause an increase in $4 \mathrm{EBP} 1$ levels. These results were confirmed by immunofluorescence staining for p-Thr37/46 4EBP1 (Figure 7A).

In order to correlate signaling alterations with changes in cytokine production, we measured cytokine protein levels and mRNA in Torin1-treated BMDMs. As shown in Supplemental Figure 8, Torin 1 treatment caused a decrease in cytokine protein levels (Supplemental Figure 8A), whereas mRNA levels remained unaltered or were upregulated (Supplemental Figure 8B), further indicating that Torin1 treatment and genetic loss of Rheb1 largely overlapped in terms of phenotypic outcome.

Phosphorylation of 4EBP1 is necessary to release the initiation factoreIF4Etostarttranslation(33). Reduced4EBP1phosphorylation at Thr37/46 and increased protein levels suggested that the defect in Rheb-KO microglia and macrophage cytokine production was due to a block in translation caused by persistent binding of $4 \mathrm{EBP} 1$ to eIF4E, inhibiting its interaction with eIF4G and therefore translation initiation. In order to test whether eIF4E and eIF4G binding was impaired in the absence of RHEB1, we carried out a proximity ligation assay, which is able to detect proteins only when in close proximity to each other (schematic, Figure 7B), in primary microglia cultures stimulated with LPS. Staining for eIF4E and eIF4G showed significantly fewer proximity ligation assay dots/ cell in Rheb-KO versus WT microglia (Figure 7B), indicating diminished interaction of eIF4E with eIF4G in the absence of RHEB1. This was not due to diminished eIF4E and eIF4G protein levels, 
A

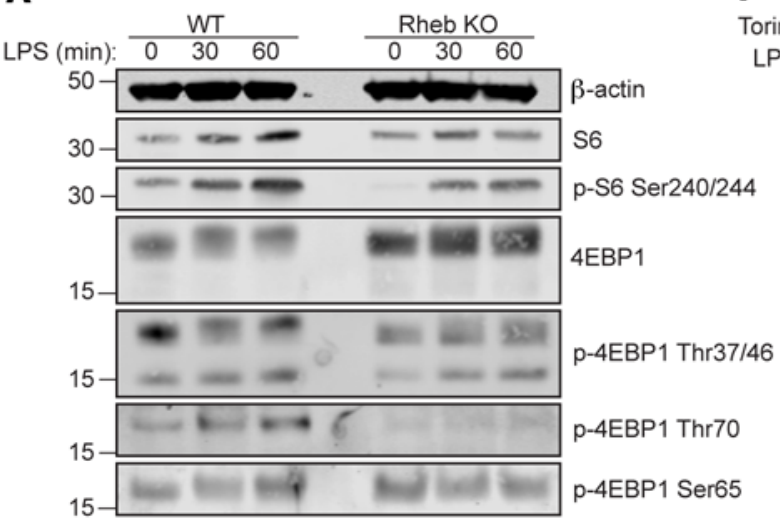

$\mathbf{F}$

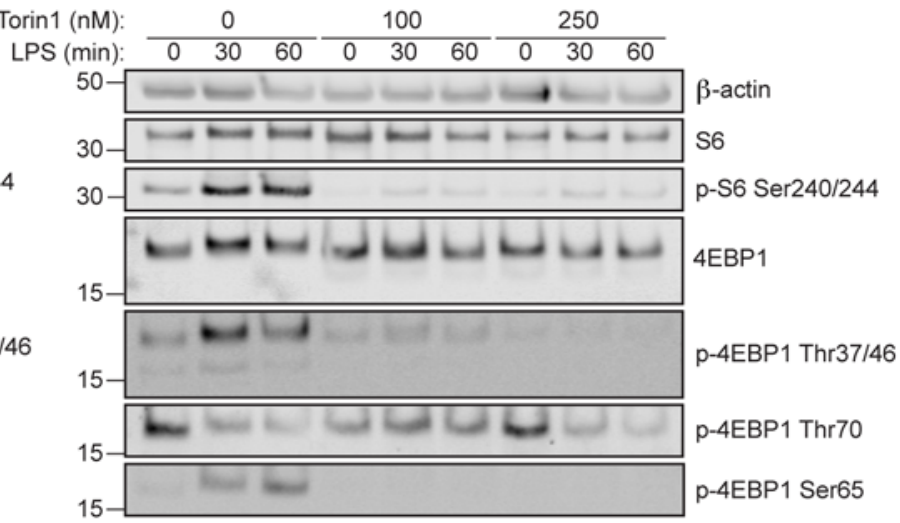

G

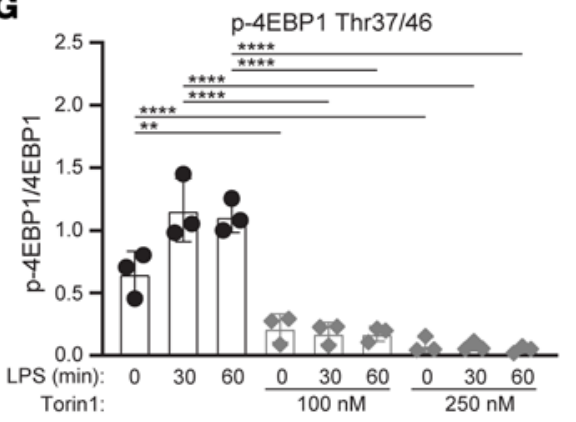

H

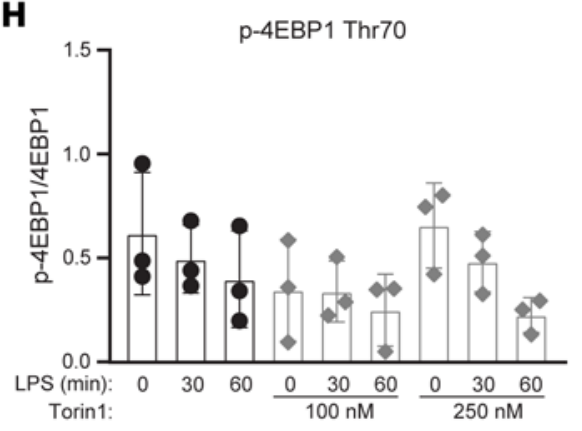

I

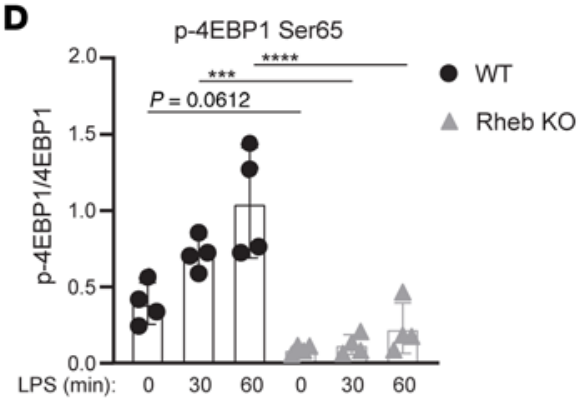

E
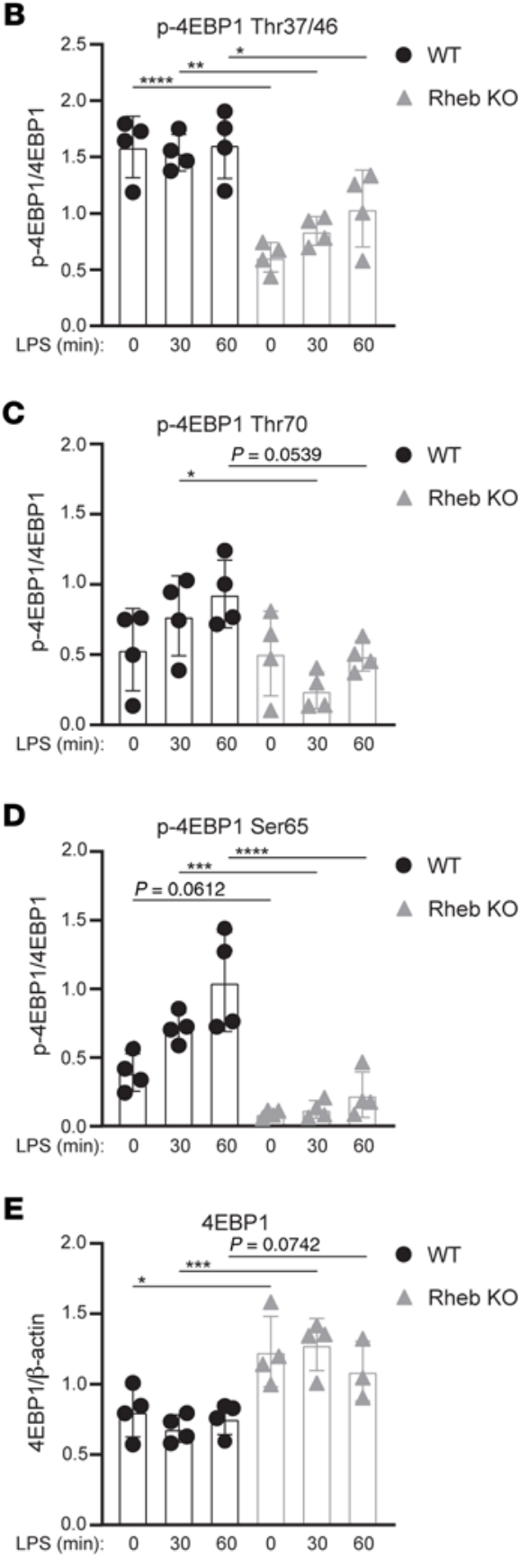

C

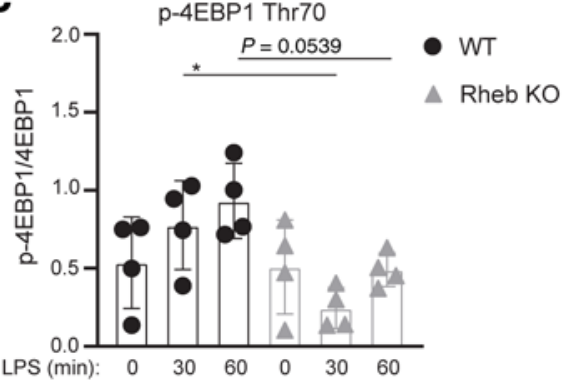

D

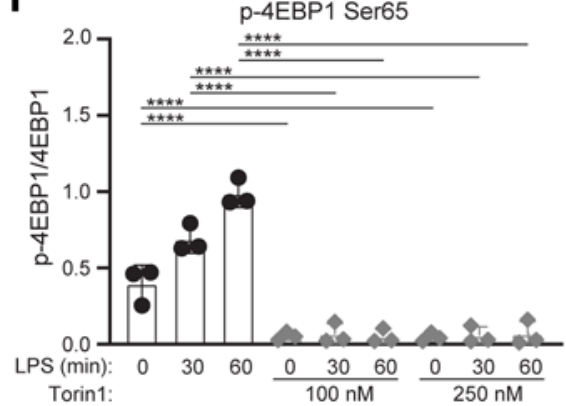

J

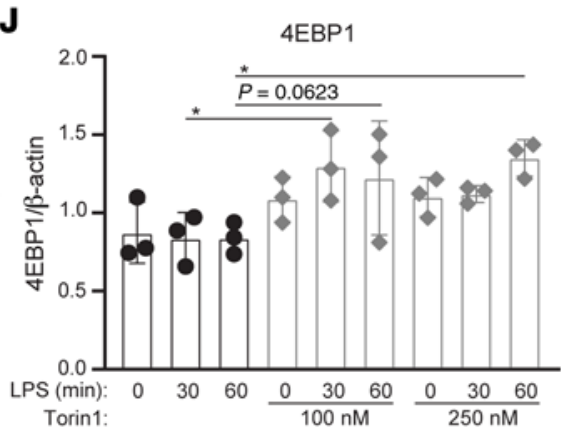

Figure 6. Rheb-KO and Torin1 exert similar effects on 4EBP1 phosphorylation. (A) Western blot of WT and Rheb-KO BMDMs stimulated with $100 \mathrm{ng} / \mathrm{mL}$ LPS for the indicated time points. (B-E) Densitometric analysis of (B) $p$-4EBP1 Thr37/46, (C) p-4EBP1 Thr70, (D) p-4EBP1 Ser65, and (E) 4EBP1 $(n=4)$. (F) Western blot of WT BMDMs pretreated with either $100 \mathrm{nM}$ or 250 nM Torin1 for 30 minutes and then stimulated with $100 \mathrm{ng} / \mathrm{mL}$ LPS for the indicated durations. (G-J) Densitometric analysis of (G) $p$-4EBP1 Thr37/46, (H) p-4EBP1 Thr70, (I) p-4EBP1 Ser65, and (J) 4EBP1 $(n=3)$ 56 and $\mathrm{p}-\mathrm{S6}$ are shown to confirm mTORC1 inhibition. ${ }^{*} P<0.05,{ }^{* *} P<$ $0.01,{ }^{* * *} P<0.001,{ }^{* * * *} P<0.0001$; 2-way ANOVA. 
A
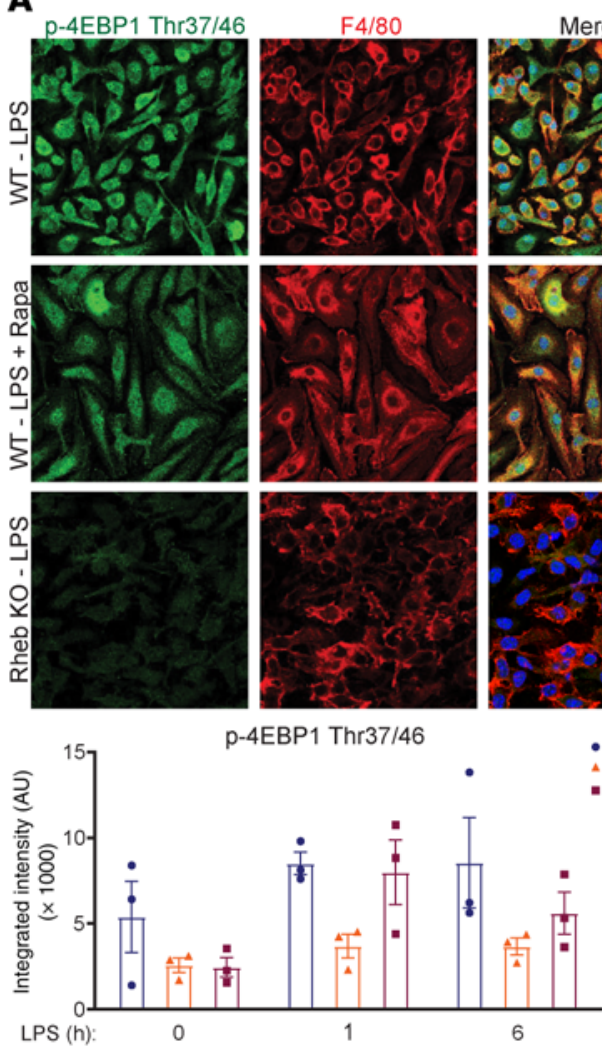

C
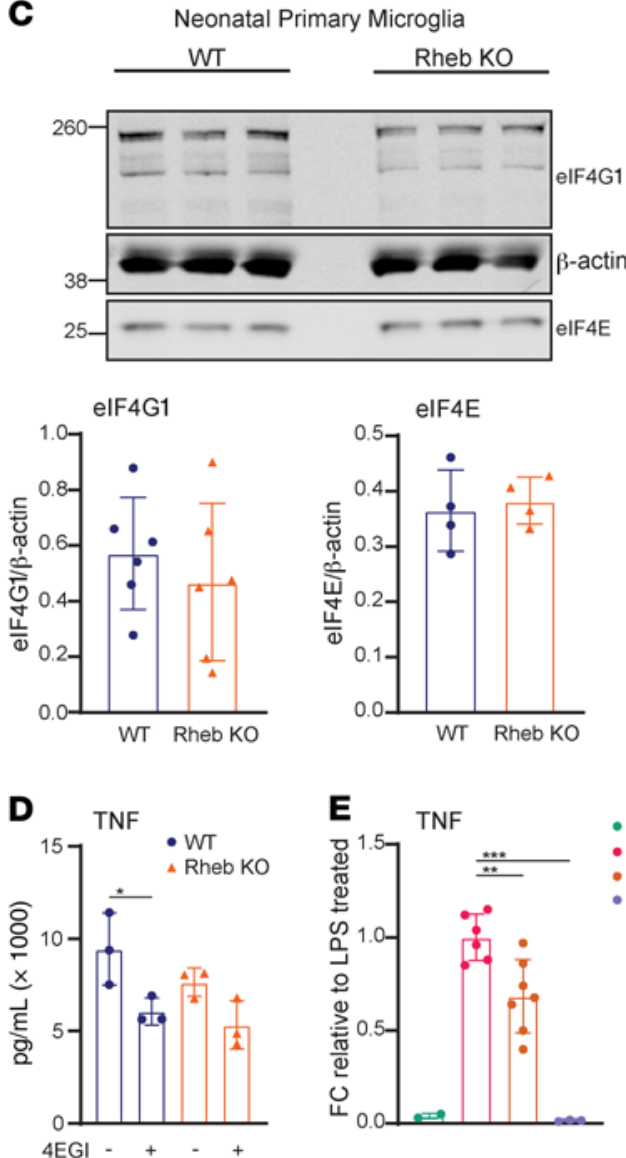

B
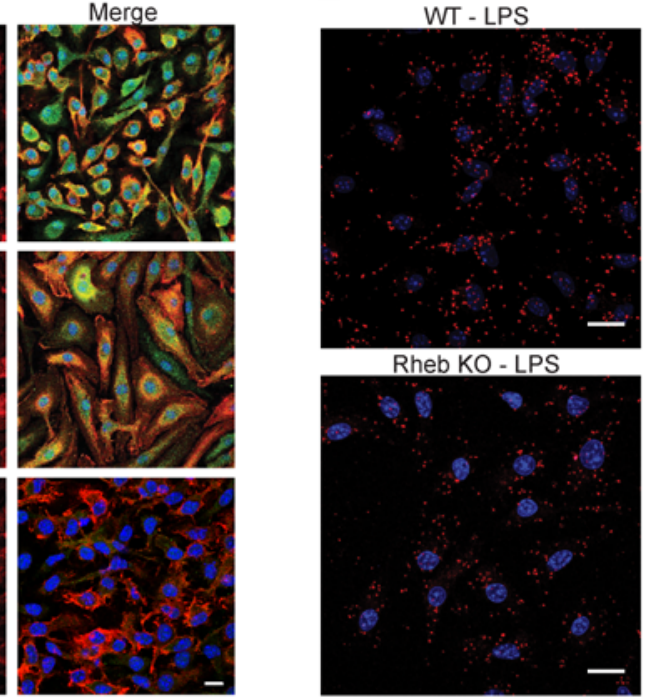

WT
Rheb KO

Rapamycin

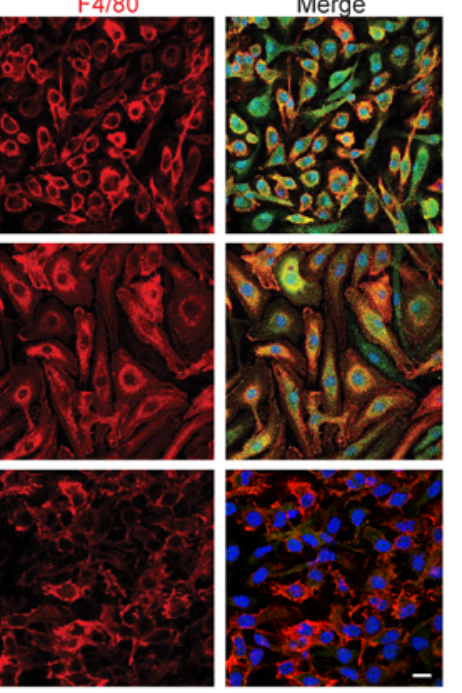

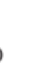

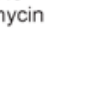
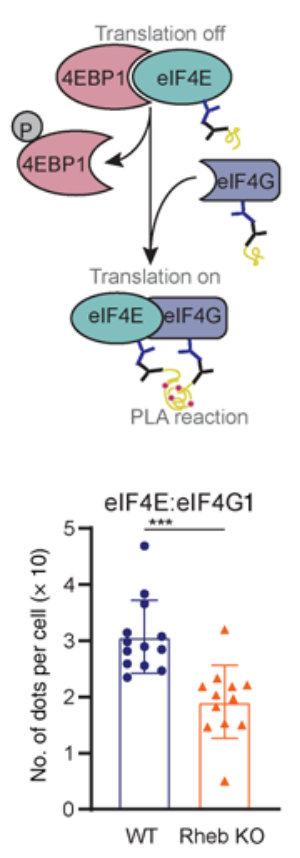

BMDMs
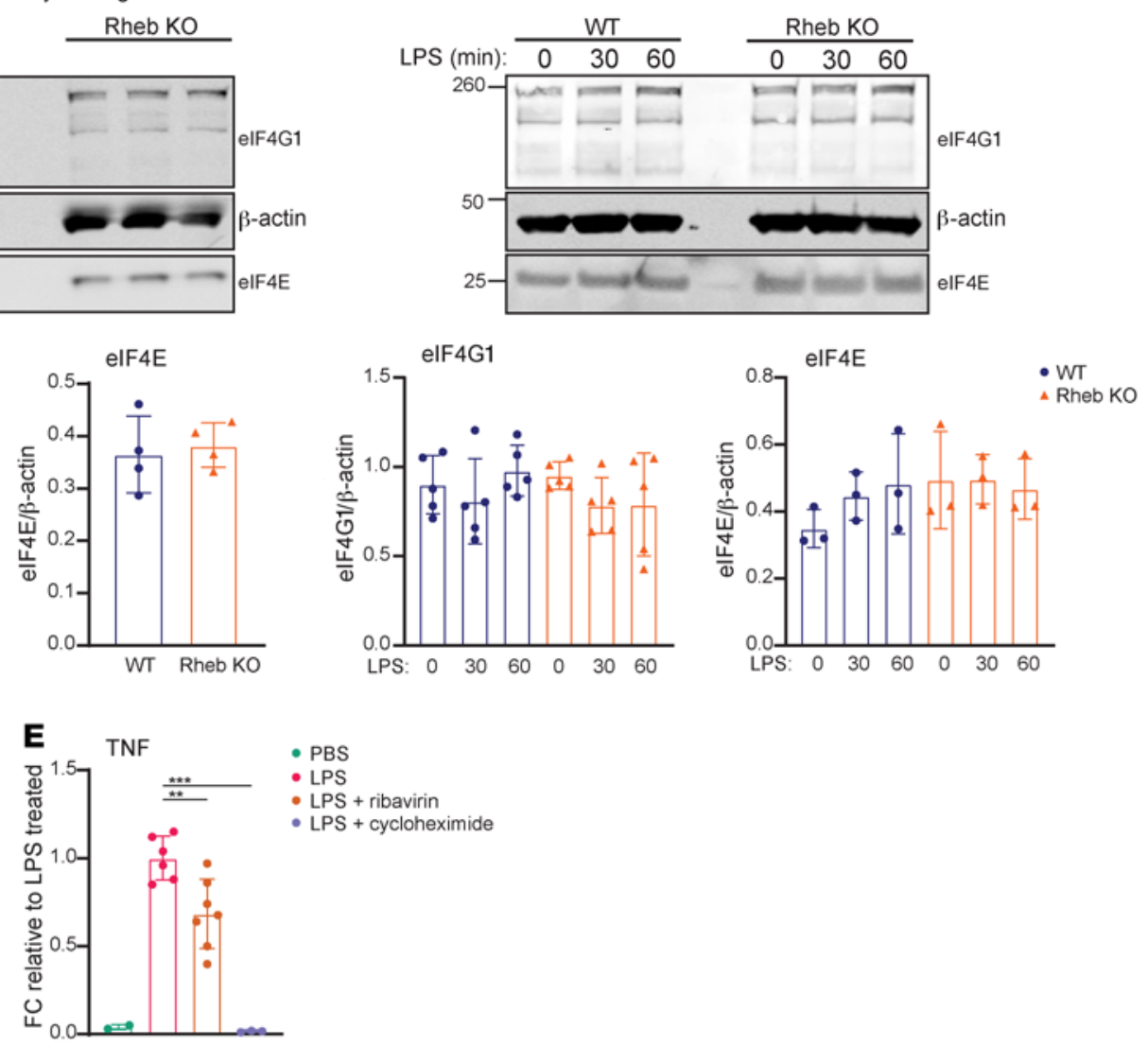
Figure 7. In Rheb-KO cells, diminished binding of elF4E to its partner elF4C results in diminished translation of cytokines. (A) Immunofluorescence staining of p-4EBP1 Thr 37/46 (green) and F4/80 (macrophage marker, red) in WT, Rheb-KO, or WT BMDMs pretreated for 90 minutes with $100 \mathrm{nM}$ rapamycin and stimulated with $100 \mathrm{ng} / \mathrm{mL}$ LPS for 1 hour (not shown) and 6 hours. Scale bar: $10 \mu \mathrm{m}$. Bottom panel: p-4EBP1 Thr37/46 quantification $(n=3)$. (B) Proximity ligation assay (PLA) of elF4E and elF4G1 in neonatal primary microglia cultures from WT and Rheb-KO brains. The schematic represents mTOR modulation of translation and the principle of PLA. Bottom panel: Quantification of elF4E/elF4C binding (PLA events, red dots). Scale bar: $25 \mu \mathrm{m}$ (objective $40 \times$ ). $n=3$ biological replicates, each with 4 technical replicates. (C) Western blot and densitometry analysis of elF4E and elF4G1 from WT and Rheb-KO microglia ( $n=6$ for elF4G1, $n=4$ for elF4E) and from WT and Rheb-KO BMDMs stimulated with $100 \mathrm{ng} / \mathrm{mL}$ LPS for the indicated time points ( $n=5$ for elF4G1, $n=3$ elF4E). (D) TNF measured by ELISA of culture supernatant from WT and Rheb-KO primary microglia. Cells were pretreated for 90 minutes with $25 \mu \mathrm{M}$ of the elF4E/elF4C inhibitor 4EGI-1 and then stimulated with $200 \mathrm{ng} / \mathrm{mL}$ LPS for 3 hours $(n=3)$. (E) TNF measured by ELISA of culture supernatant from acute brain slices of WT mice, pretreated for 2 hours with either $100 \mu \mathrm{M}$ ribavirin, $40 \mu \mathrm{M}$ cycloheximide, or vehicle and then stimulated with $2 \mu \mathrm{g} / \mathrm{mL}$ LPS for 6 hours. Data are represented as the fold change normalized to LPS samples; $n=6$ from 2 independent experiments. ${ }^{*} P<0.05,{ }^{*} P<0.01,{ }^{* *} P<0.001$; 2-way ANOVA (A, C [BMDMs], and D; Student's $t$ test (B, C [microglia], and E). For $\mathbf{A}, P=0.0041$ for WT versus Rheb-KO and NS $(P=0.1465)$ for WT versus rapamycin; 2-way ANOVA.

which were comparable between WT and Rheb-KO cells, in microglia and macrophages (Figure $7 \mathrm{C}$ ). In order to confirm that the decreased binding of eIF4E to eIF $4 \mathrm{G}$ was the critical event responsible for the Rheb-KO phenotype, we used the pharmacological inhibitor 4EGI-1, which disrupts the binding of eIF4E to eIF $4 G$ and consequently inhibits translation $(64,65)$. Pretreatment of WT microglia with $4 \mathrm{EGI}-1$ led to a reduction of TNF protein to a level comparable to Rheb-KO microglia; however, it did not cause a further reduction in Rheb-KO microglia (Figure 7D). Additional cytokines/chemokines were also diminished by 4EGI-1 in WT cells but were already downregulated in Rheb-KO cells (Supplemental Figure 7C). This further confirmed that eIF4E/eIF4G binding was already impaired in the absence of RHEB1, and it was responsible for the observed effect on cytokine translation. Accordingly, eIF4E phosphorylation at Ser209 was also impaired (Supplemental Figure 7D). This phosphorylation is mediated by ERK-activated kinases MNK1 and MNK2 (66-68); nonetheless, it requires eIF4E to be bound to eIF4G because eIF4G functions as a docking site for MNKs $(66,69)$. The defect in eIF4E Ser209 phosphorylation correlated with partially reduced ERK activation (Supplemental Figure 7E). Taken together, these results further confirmed that Rheb-KO cells had an impairment in mRNA translation due to diminished activation of eIF4E.

In order to assess whether it was possible to therapeutically target eIF4E to modulate brain inflammatory responses, we treated acute brain slices with the eIF4E inhibitor ribavirin, an antiviral drug $(43,70)$. By treating acute brain slices from WT mice, we found that ribavirin significantly reduced TNF levels in the culture media after LPS treatment (Figure 7E), whereas treatment with cycloheximide completely inhibited TNF production (Figure 7E), further indicating that eIF4E inhibition exerts a subtler control over translation of the inflammatory response. Taken together, our data indicate that binding of eIF4E to eIF4G regulated translation of inflammatory mediators in microglia and could represent a novel therapeutic target in neuroinflammation.

An $m$ TOR/translational signature is present in aging human microglia and in damage-associated microglia from mouse models of neurodegenerative diseases. In order to assess whether the mTOR translational signature found in microglia from aging mice was also present in aging human microglia, we mined publicly available data sets. By bioinformatics analysis of the Olah et al. data set (71), which assessed gene expression changes in aging human microglia, we found that of the mTOR- and translation-related genes upregulated in aged mouse microglia, $62.5 \%$ were also upregulated in human microglia from old brains (Figure 8A). Interestingly, the fold changes in human aging microglia were about 5-8 times higher than in mouse counterparts. A similar proportion of genes $(57.1 \%)$ was upregulated in a neurodegeneration-specific microglia population or damage-associated microglia identified in mouse models of amyloid- $\beta$ deposition (72) (Figure 8B). In particular, genes coding for ribosome subunits were strongly upregulated in both aging and damage-associated microglia (data not shown), indicating that upregulation of translation is a common mechanism that occurs in aging and in neurodegeneration across species and could constitute an important therapeutic target.

\section{Discussion}

Microglia have been implicated in the pathogenesis of neurodegenerative diseases, fueling their initiation and progression, especially through a heightened ability to respond to activation stimuli, also called priming $(73,74)$. Microglia appear primed with age and in neurodegenerative diseases; however, little is known about what causes these changes. A wealth of RNA-Seq and single-cell RNA-Seq data have greatly increased our knowledge of microglia in health and disease. However, these studies define microglia mostly through their transcriptome profiles. Our work has highlighted an important difference between young and aged microglia: translation in aged microglia is increased because of an increase in the mTOR pathway. This means that transcriptional profiling could be missing crucial aspects of microglia biology in aging as well as neurodegeneration.

The upregulation of mTOR signaling and translation could be caused by intrinsic changes in microglia or by the aging neural environment. Raj et al. showed that neuronal deletion of Ercc1, a DNA excision repair protein, was sufficient to switch microglia to a primed phenotype, suggesting that neuronal genotoxic stress is a contributing factor (75). Furthermore, mTOR is a pivotal sensor of growth factors (76). Interestingly, one of the genes associated with the priming phenotype in our data set was insulin-like growth factor 1 (Igfl) also found by others (13). In accordance with those studies, we found the protein IGF1 to be significantly increased in the aging brain, despite systemic levels declining with age (77). Therefore, it is possible that microglia or other cells in the brain upregulate IGF1 with age, leading to the hyperactivation of mTOR. Other growth factors increase in the aging brain, such as brain-derived neurotrophic factor (BDNF) and nerve growth factor (NGF) (78), although we did not observe an upregulation in NGF in our brain lysates and BDNF was not measured. Regardless of whether one or multiple factors regulate $\mathrm{mTOR}$ signaling in aged microglia, it will be interesting to define which cells produce them, in response to what alterations, 
A
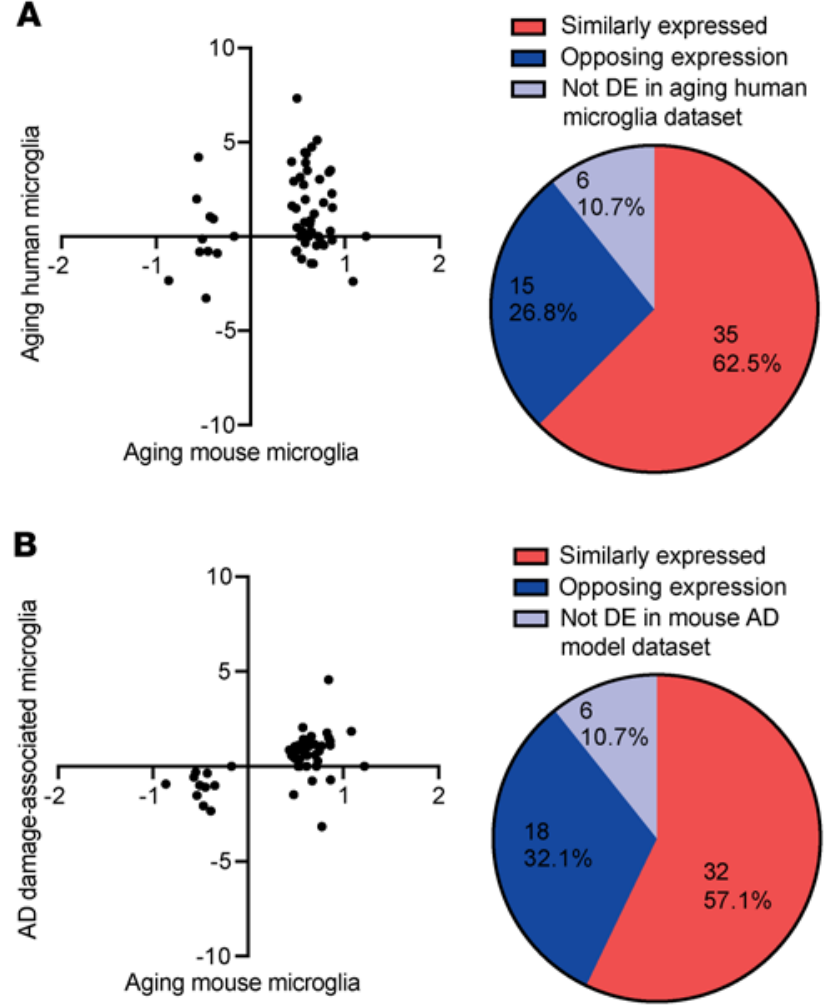

Figure 8. A similar signature for increased translation is present in aging human microglia and damage-associated microglia. (A) Left panel: $\log _{2}$ fold changes for elF2 signaling, mTOR signaling, and regulation of elF4 and p70K signaling pathway genes in aging mouse ( $x$ axis) and human microglia (y axis). Right panel: Comparison of differentially expressed genes within the 3 pathways. (B) Left panel: $\log _{2}$ fold changes for elF2 signaling, mTOR signaling, and regulation of elF4 and p70K signaling pathway genes in aging mouse microglia ( $x$ axis) and damage-associated microglia ( $y$ axis). Right panel: Comparison of differentially expressed genes within the 3 pathways. DE, differentially expressed.

and whether a similar upregulation takes place in neurodegenerative diseases. Aged microglia share many features with damageassociated microglia from Alzheimer disease and amyotrophic lateral sclerosis at the transcriptional level (8). Our bioinformatics analysis suggests that altered translation might be shared by aged and damage-associated microglia; therefore, future studies should focus on whether increased translation in an inflammatory response contributes to microglia becoming more neurotoxic.

In terms of mTOR-regulation of translation, our work further highlightsthatalthoughmTORisknowntoregulatecap-dependent translation, it does not regulate all mRNAs equally $(47,79)$. Indeed, housekeeping genes encoding for $\beta$-actin or GAPDH, for example, are not as sensitive as genes encoding proteins involved in the immune response, such as SPP1, VEGF, MMP9, IRF7, and IкB- $\alpha(38,79-81)$. It is believed that specific characteristics in the mRNA confer increased eIF4E (and therefore mTORC1) sensitivity. Nonetheless, these motifs appear to differ in different cell types $(39,40,44,56,82)$. Originally, a long $5^{\prime}$-UTR, rich in secondary structures, was thought to confer mTORC1 specificity $(79,80)$; however, more recently, it has emerged that translation of mRNAs without a long 5 '-UTR can still be sensitive to eIF4E $(42,82)$. Furthermore, additional features have been described, such as $5^{\prime}$ terminal oligopyrimidine tracts (TOPs or TOP like) (44) and CERT, cytosine-rich 15-nucleotide motif (39). It is possible to speculate that priming and inflammatory genes in microglia could also share a yet unidentified consensus sequence because the majority of the genes we investigated did not show any of these known ones (Supplemental Table 4). An in-depth study of mTOR-dependent genes in microglia could potentially identify new motifs and shed more light on how mTOR modulation would affect microglia inflammatory responses.

In terms of pharmacological regulation of mTOR-signaling controlling translation, we observed that rapamycin did not affect 4EBP1 phosphorylation at Thr37/46, whereas the ATP-competitive inhibitor Torin1 (56) significantly impaired it. This difference correlated with a differential regulation of $4 \mathrm{EBP} 1$ protein levels: rapamycin did not cause any alteration, whereas Torin1 treatment caused an upregulation in a similar fashion to Rheb-KO cells. This difference could explain why translation of inflammatory genes is not impaired after rapamycin treatment, as described by Weichhart et al. (21); however, it was impaired upon Torin1 treatment or in Rheb-KO cells. The correlation between decreased Thr37/46 phosphorylation and increased 4EBP1 protein levels is consistent with a previous report showing that $4 \mathrm{EBP1}$ phosphorylation, in particular at $\mathrm{Thr} 37 / 46$, leads to $4 \mathrm{EBP} 1$ ubiquitination and subsequent degradation (83). Our results have important implications for the development of new drugs targeting mTOR. On the one hand, the results suggest that mTOR regulation of translation is a valid therapeutic target, especially to prevent increased neuroinflammation linked to aging; on the other hand, they illustrate the importance of fully understanding how mTOR regulates inflammatory processes at multiple levels before designing new pharmacological modulators.

There is extensive literature on the consequences of mTOR therapeutic inhibition. The best-known effect mediated by rapamycin is lifespan extension in lower organisms and mammals, mostly attributed to mTOR's function as a nutrient sensor $(84,85)$. In addition, rapamycin counteracts the progression of neurodegenerative diseases and age-related cognitive decline through modulation of autophagy in neurons and prevention of the accumulation of misfolded proteins $(28,29,86)$. Additionally, mTOR inhibition leads to altered $\mathrm{T}$ and $\mathrm{B}$ cell differentiation that favor immune tolerance, resulting in rapamycin analogs being used in transplantation (87). However, there are also reports that rapalogs cause potentially deleterious proinflammatory effects, for example in transplant patients, by increasing the inflammatory phenotype of myeloid cells (88). In line with this evidence, rapamycin analogs administered shortterm resulted in improved vaccination responses in the elderly, possibly through increased myeloid activation $(31,89)$. Therefore, the literature indicates that modulation of mTOR with different pharmacological inhibitors, as well as duration of treatment, might cause apparently contradictory results. The more we learn about mTORC1- (and mTORC2-) dependent functions, the more we will be able to design targeted therapeutic strategies.

Indeed, our study adds to our knowledge of the mTOR pathway in inflammation by elucidating the effect of Rheb1 loss in microglia and the immune system. Rheb1 loss did not cause significant alterations in the immune system at the steady state, consistently with previous reports using Rheb $1^{A / f l} R O S A 26-E R T 2^{\mathrm{Cre}}(90)$. This might be due to residual mTORC1 signaling in KO cells. Whether this 
signaling is due to Rheb-independent mTORC1 activity or incomplete loss of Rheb1 in some cells remains to be further elucidated. The partial inhibition of mTORC1 activity in Rheb1 $1^{f / f l}$ Csflr ${ }^{\text {Cre }}$ has its advantages, as it allowed us to assess microglia inflammatory responses in young and aged animals: a stronger inhibition could have caused too great an impairment in cell differentiation and survival. Nonetheless, it offers an additional reminder that different pharmacological inhibitors of mTOR might cause different outcomes, based on mode of action and duration of the inhibition.

\section{Methods}

Mice. Aged female C57BL/6J mice were obtained from Charles River Laboratories. For RNA-Seq experiments, ex-breeder female mice were used at 6,15 , and 23 months of age. Mice with a transgenic construct containing a Cre recombinase coding sequence under the control of the promoter for the murine colony stimulating factor 1 receptor $\left(C s f 1 r^{C r e}\right)$ were obtained from The Jackson Laboratory. Mice with loxP-flanked Rheb1 exon 3 were generated in the laboratory of PF Worley, Johns Hopkins University School of Medicine, Baltimore, Maryland, USA (51) and were provided as a gift. Exon 3 encodes the critical GTP binding domain; its deletion causes a frameshift mutation and consequent loss of RHEB1 protein.

Adult microglia isolation. The brain and spinal cord were perfused with ice-cold HBSS and tissue was digested using the Neural Tissue Dissociation Kit from Miltenyi Biotec. Cells were washed twice in HBSS and myelin was removed by negative selection using myelin removal beads (Miltenyi Biotec, 130-096-433). For RNA-Seq experiments, cells were then incubated with microglia antibodies CD11b and CD 45 for 20 minutes at $4^{\circ} \mathrm{C}$ in the dark and then flow-sorted on an LSR-Fortessa (BD Biosciences). DAPI (Cambridge Bioscience) was used to exclude dead cells. For all other experiments, microglia were isolated after myelin removal by positive section using CD11b beads (Miltenyi Biotec, 130-093-634).

RNA extraction, assessment, and sequencing. For RNA-Seq experiments, microglia were sorted from 3 separate mice per replicate and pooled prior to RNA extraction, which was carried out using an RNeasy Micro Kit (Qiagen), according to the manufacturer's guidelines. Briefly, cells were lysed in Qiagen RLT lysing buffer before an equal volume of $70 \%$ ethanol was added to the lysates, which were placed on a silicon column and washed 3 times with RW1 buffer, RPE wash buffer, and finally with $80 \%$ ethanol. RNA was then eluted using nuclease-free water and quantified using a Nano Drop 1000 (Thermo Fisher Scientific). The quality and quantity of RNA was assessed by the Agilent Bioanalyzer 2100 RNA Pico Chip. Only samples with a RNA integrity number (RIN) greater than 7 were subjected to RNA-Seq. RNA samples were processed by Oxford Gene Technology (OGT), where they were prepared for RNA-Seq using the SMARTer Ultra Low RNA kit for Illumina sequencing (Clontech Laboratories). RNA-Seq libraries were then sequenced on the Illumina HiSeq 2000, achieving approximately 40 million paired-end reads per sample. RNA-Seq data have been deposited in NCBI's GEO and Sequence Read Archive (SRA) databases with the accession number GSE156762.

RNA-Seq data analysis. Raw sequencing reads were demultiplexed by OGT. FastQC (version 0.11.3; ref. 91) was used to assess sequencing read quality. Reads were mapped to the mouse genome (GRCm38.p5, GENCODE release M14; refs. 92, 93) using STAR (version 2.5.3 with option-outFilterIntronMotifs RemoveNonCanonical; ref. 94) with default parameters, and only uniquely mapping reads were selected for further analysis. Ribosomal RNA and mitochondrial reads were removed using modified scripts from the PORT pipeline (https:// github.com/itmat/Normalization). SAM files were converted to BAM files using Samtools view and BAM files were sorted by coordinate with Samtools sort. Annotations were based on the comprehensive gene annotation file of the GENCODE Release M14 (mm10). The downloaded Gene Transfer Format (GTF) file was loaded into R (version 3.4.0; R Foundation for Statistical Computing) and converted into a TranscriptDb object with the makeTranscriptDbFromGFF tool in the Bioconductor package GenomicFeatures (version 1.28.3; ref. 95). From the TranscriptDb object, all annotated Ensembl genes and their exons were obtained using exonsBy (by = "gene"). Ensembl gene IDs were replaced by official gene symbols using biomaRt (version 2.32.1). Genes with several Ensembl gene IDs were combined into 1 record. For each duplicated gene, overlapping exons were combined into single exons. Thus, genes were defined as the sequence between the first base of the first exon and the last base of the last exon. Furthermore, regions shared by overlapping genes were removed because we worked with a nonstranded RNA-Seq library and wanted to count only reads mapping to 1 gene. The mapped, filtered RNA-Seq reads were counted using a custom R script, including the R packages Rsamtools (1.22.0), GenomicFeatures (1.22.8), and GenomicAlignments (1.6.3; ref. 95). Briefly, sorted BAM files were loaded into R using readGAlignmentPairs, and the number of reads mapping to genes was computed using findOverlaps (with options type = "within" and ignore.strand = TRUE) and countSubjectHits. Genes were analyzed for differential expression using the R package DESeq2 (1.16.1; ref. 96) and identified as differentially expressed if the FDR-adjusted $P$ value was smaller than 0.05 . PCA plots and heatmaps were plotted using R. Gene abundances were measured by DESeq2's rlog transformed median of ratios. Counts were divided by sample-specific size factors determined by median ratio of gene counts relative to geometric mean per gene, which normalizes for sequencing depth and RNA composition. PCA was then performed based on the top 500 most variable genes across the 9 samples to determine their spatial distances between each other (i.e., verify the biological groups based on RNA-Seq data). Canonical pathway enrichment analysis was further conducted based on the identified differentially expressed genes using the IPA tool (Qiagen), thanks to its most comprehensive curated annotation resources.

Protein synthesis assay. At day 6 of BMDM differentiation, 50,000 cells were plated in 96-well clear bottom, black, cell culture plates and allowed to adhere overnight. The following day, BMDMs were stimulated with $100 \mathrm{ng} / \mathrm{mL}$ LPS for 1 hour or 3 hours. Samples were further processed according to the manufacturer's instructions using the Protein Synthesis Assay Kit (Cayman Chemical, 601100). Briefly, cells were treated for 2 hours at $37^{\circ} \mathrm{C} / 5 \% \mathrm{CO}_{2}$ with $100 \mu \mathrm{L}$ DMEM as control, O-propargyl-puromycin (OPP) working solution, and/ or OPP working solution with cycloheximide (1:1,000). Fluorescence intensity was measured with a fluorescent plate reader using a 485/520 nm filter (FLUOstar Omega, BMG Labtech).

In vivo LPS. For in vivo experiments, mice were i.p. injected with either $200 \mu \mathrm{L}$ E. coli 0111:B4 (Sigma-Aldrich, catalog no. L2630, EC no. 297-473-0) or PBS as control. The dose of LPS was $5 \mathrm{mg} / \mathrm{kg}$ and mice were culled after 4 hours, except for sickness behavior experiments where a lower dose of $0.33 \mathrm{mg} / \mathrm{kg}$ was used. For aging experiments, mice used were 2-6 months (young) or 14-18 months (old) of age. For experiments with Rheb1 $1^{f / f l}$ Csflr ${ }^{\text {Cre }}$ mice, male and female mice were used at 2-6 months (young) and 


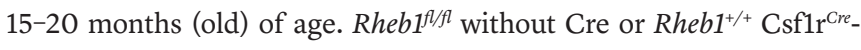
positive mice were used as WT controls.

Proximity ligation assay. Primary microglia were generated from PO-P3 WT and Rheb-KO pups as described in Supplemental Methods and treated with $100 \mathrm{ng} / \mathrm{mL}$ LPS for 3 hours. After LPS treatment, cells were fixed for 10 minutes with $4 \%$ PFA and washed 3 times with PBS. Cells were then permeabilized using 0.5\% Triton X-100 (Sigma-Aldrich) for 15 minutes and then blocked for 1 hour at room temperature using mouse on mouse blocking reagent (Vector Laboratories, MKB-2213), followed by an additional blocking stage using the Duolink blocking solution (Sigma-Aldrich, DUO92101) for 1 hour at $37^{\circ} \mathrm{C}$. Cells were then incubated overnight at $4^{\circ} \mathrm{C}$ in anti-mouse eIF4E (Invitrogen, MA1-089, 1:200) or anti-rabbit eIF4G (Cell Signaling Technology, 2469, 1:1000). The next day, cells were incubated in anti-mouse plus and anti-rabbit minus proximity ligation assay probes (Sigma-Aldrich, DUO92101) diluted 1:5 in the provided antibody diluent for 1 hour at $37^{\circ} \mathrm{C}$. Cells were then washed twice with wash buffer A for 5 minutes at room temperature, and then the ligase (prediluted in ligation buffer 1:40) was added to cells for 30 minutes at $37^{\circ} \mathrm{C}$. This step was followed by further washing with wash buffer A, prior to addition of the DNA polymerase (diluted in amplification buffer, $1: 80$ ) for 100 minutes at $37^{\circ} \mathrm{C}$. Subsequently, cells were washed using wash buffer B, then Duolink in situ mounting media containing DAPI (Sigma-Aldrich, DUO82040) was used for mounting coverslips. All samples were analyzed using a confocal microscope (LSM710, Zeiss) within 48 hours of preparation.

Statistics. For RNA-Seq experiments, significance of the differential expression of genes was evaluated based on FDR-adjusted $P$ values smaller than 0.05 calculated by the R package DESeq2. The Qiagen IPA tool used the Benjamini and Hochberg correction for multiple comparisons (97). To judge significance in other experiments, Student's $t$ test, 1-way ANOVA, 2-way ANOVA, and 3-way ANOVA analyses were performed using GraphPad Prism. A 2-tailed, unpaired Student's $t$ test was used to calculate significance between 2 groups or populations. Depending on the number of categorical independent values per experiment, 1-way, 2-way, or 3-way ANOVA approaches were used to judge the impact of each independent category on the experimental outcome. In combination with ANOVA, the Šídák multiple-comparison test (98) was used for the hypothesis-driven calculation of multiple-comparison corrected $P$ values (reported in the graphs). In aging experiments with more than 2 time points and 1 categorical independent value, the linear trend was also analyzed. All data are reported with error bars representing the standard error of the mean. All $P$ values less than 0.05 were considered significant and indicated with 1-4 asterisks: ${ }^{*} P<0.05,{ }^{* *} P<0.01,{ }^{* * *} P<0.001$, and ${ }^{* * * *} P<0.0001$. Close-to-significance comparisons were indicated with the actual $P$ value. Nonsignificant differences are indicated by NS. Gray bars and asterisks indicate comparison within the same age group or the same genotype. Black bars and asterisks indicate comparison across different age groups or across different genotypes. Statistical analysis results for all panels shown are provided in Supplemental Table 5. Data were reported as acquired raw data whenever possible. In certain instances, in order to allow statistical analysis on pooled experiments repeated on separate days, data were expressed as fold change relative to control.

Study approval. Mice were housed according to UK Home Office and German NRW Landesamt für Natur, Umwelt und Verbraucherschutz Nordrhein-Westfalen (LANUV NRW) guidelines. All exper- iments involving mice were conducted in accordance with and approved by the UK Home Office license PPL 70/7411 and German LANUV NRW license 81-02.04-2018.A257.

\section{Author contributions}

LK and MC conceived the study, designed experiments, interpreted data, and wrote the first draft of the manuscript. LK and RZ carried out the RNA-Seq experiment and RZ carried out a first analysis of the results, with help from AN, CC, and JW. The data set was reanalyzed by SPR and JR, who carried out further bioinformatics analysis and comparisons with published data sets. LK carried out the majority of the experiments described, and IA performed experiments concerning protein synthesis, cell signaling, autophagy, rapamycin, and Torin1 and helped perform in vivo experiments. SPR ran the statistical analysis for most experiments, with help from IA. SPR also performed qPCR on Torin1-treated samples and analyzed results. Both IA and SPR significantly contributed to the preparation of the manuscript. AAD carried out the staining for p65-RelA and p-4EBP1, as well as ELISA and qPCR for the rapamycin experiments. NO performed the TNF staining on brain tissue and helped perform in vivo experiments. MEB carried out the LC3 staining on brain tissue and quantification and IBA1 staining on young brains with relative quantification and helped perform in vivo experiments. FG performed staining and quantification of IBA1 staining on young and old brains. PNM performed a repeat of the open-field test. JE contributed to tissue culture experiments and immunofluorescence staining. YB contributed to experiments with acute brain slices. DT, AH, PS, and MTH assisted with data interpretation, provided reagents, and critically reviewed the manuscript. All authors read and approved the manuscript. LK is listed before IA as first author, as she played a greater role in conceiving the study and performing experiments.

\section{Acknowledgments}

The authors thank PF Worley, Johns Hopkins University School of Medicine, Baltimore, Maryland, USA, for his gift of the Rheb1-floxed mouse line. We also gratefully acknowledge the animal technical service at the Barts Cancer Institute, Queen Mary University of London, UK, in particular the contribution of J. Andow-Cleaver and C. Pegrum. This work was supported by Cancer Research UK core funding to Barts Cancer Centre (C16420/A18066), AgeUK (PhD studentship to LK, grant 375); the Dunhill Medical Trust (grant R475/0216), and Rosetrees Trust (grant JS16/M513). MC was supported by a Bennett Fellowship from Bloodwise (grant 12002) and by DZNE core funding. This work was also supported by the Helmholtz-Gemeinschaft, Zukunftsthema Immunology and Inflammation (ZT-0027) and by the Deutsche Forschungsgemeinschaft (DFG, German Research Foundation) under Germany's Excellence Strategy EXC2151-390873048.

Address correspondence to: Melania Capasso, German Center for Neurodegenerative Diseases (DZNE), Venusberg-Campus 1, Gebäude 99, 53127 Bonn, Germany. Email: melania.capasso@ dzne.de. LK's present address is: Institute of Environmental Medicine, Toxicology Unit, Karolinska Institutet, Stockholm, Sweden. 
1. Hanisch UK, Kettenmann H. Microglia: active sensor and versatile effector cells in the normal and pathologic brain. Nat Neurosci. 2007;10(11):1387-1394.

2. Harry GJ. Microglia during development and aging. Pharmacol Ther. 2013;139(3):313-326.

3. Kettenmann H, Hanisch UK, Noda M, Verkhratsky A. Physiology of microglia. Physiol Rev. 2011;91(2):461-553.

4. Kreutzberg GW. Microglia: a sensor for pathological events in the CNS. Trends Neurosci. 1996;19(8):312-318.

5. Hoshiko M, Arnoux I, Avignone E, Yamamoto N, Audinat E. Deficiency of the microglial receptor CX3CR1 impairs postnatal functional development of thalamocortical synapses in the barrel cortex. JNeurosci. 2012;32(43):15106-15111.

6. Perry VH, Holmes C. Microglial priming in neurodegenerative disease. Nat Rev Neurol. 2014;10(4):217-224.

7. Niraula A, Sheridan JF, Godbout JP. Microglia priming with aging and stress. Neuropsychopharmacology. 2017;42(1):318-333.

8. Holtman IR, et al. Induction of a common microglia gene expression signature by aging and neurodegenerative conditions: a co-expression meta-analysis. Acta Neuropathol Commun. 2015;3:31.

9. Perry VH, Teeling J. Microglia and macrophages of the central nervous system: the contribution of microglia priming and systemic inflammation to chronic neurodegeneration. Semin Immunopathol. 2013;35(5):601-612.

10. Bachiller S, et al. Microglia in neurological diseases: a road map to brain-disease dependent-inflammatory response. Front Cell Neurosci. 2018;12:488.

11. Hickman S, Izzy S, Sen P, Morsett L, El Khoury J. Microglia in neurodegeneration. Nat Neurosci. 2018;21(10):1359-1369.

12. Wendeln AC, et al. Innate immune memory in the brain shapes neurological disease hallmarks. Nature. 2018;556(7701):332-338.

13. Hickman SE, et al. The microglial sensome revealed by direct RNA sequencing. Nat Neurosci. 2013;16(12):1896-1905.

14. Orre $\mathrm{M}$, et al. Acute isolation and transcriptome characterization of cortical astrocytes and microglia from young and aged mice. Neurobiol Aging. 2014;35(1):1-14.

15. Grabert K, et al. Microglial brain region-dependent diversity and selective regional sensitivities to aging. Nat Neurosci. 2016;19(3):504-516.

16. Byles V, et al. The TSC-mTOR pathway regulates macrophage polarization. Nat Commun. 2013;4:2834.

17. Hammond TR, et al. Single-cell RNA sequencing of microglia throughout the mouse lifespan and in the injured brain reveals complex cell-state changes. Immunity. 2019;50(1):253-271.e6.

18. Laplante M, Sabatini DM. mTOR signaling in growth control and disease. Cell. 2012;149(2):274-293.

19. Delgoffe GM, et al. The kinase mTOR regulates the differentiation of helper $\mathrm{T}$ cells through the selective activation of signaling by mTORC1 and mTORC2. Nat Immunol. 2011;12(4):295-303.

20. Powell JD, Pollizzi KN, Heikamp EB, Horton MR. Regulation of immune responses by MTOR. Annu Rev Immunol. 2012;30:39-68.

21. Weichhart T, et al. The TSC-mTOR signaling path- way regulates the innate inflammatory response. Immunity. 2008;29(4):565-577.

22. Linke $\mathrm{M}$, et al. Chronic signaling via the metabolic checkpoint kinase mTORC1 induces macrophage granuloma formation and marks sarcoidosis progression. Nat Immunol. 2017;18(3):293-302.

23. Herranz N, et al. mTOR regulates MAPKAPK2 translation to control the senescence-associated secretory phenotype. Nat Cell Biol. 2015;17(9):1205-1217.

24. Zhang HM, Diaz V, Walsh ME, Zhang Y. Moderate lifelong overexpression of tuberous sclerosis complex 1 (TSC1) improves health and survival in mice. SciRep. 2017;7(1):834.

25. Zhang HM, Fu J, Hamilton R, Diaz V, Zhang Y. The mammalian target of rapamycin modulates the immunoproteasome system in the heart. JMol Cell Cardiol.2015;86:158-167.

26. Harrison DE, et al. Rapamycin fed late in life extends lifespan in genetically heterogeneous mice. Nature. 2009;460(7253):392-395.

27. Neff $F$, et al. Rapamycin extends murine lifespan but has limited effects on aging.JClin Invest. 2013;123(8):3272-3291.

28. Malagelada C, Jin ZH, Jackson-Lewis V, Przedborski S, Greene LA. Rapamycin protects against neuron death in in vitro and in vivo models of Parkinson's disease. JNeurosci. 2010;30(3):1166-1175.

29. Spilman $P$, et al. Inhibition of mTOR by rapamycin abolishes cognitive deficits and reduces amyloid-beta levels in a mouse model of Alzheimer's disease. PLoS One. 2010;5(4):e9979.

30. Wang IF, et al. Autophagy activators rescue and alleviate pathogenesis of a mouse model with proteinopathies of the TAR DNA-binding protein 43. Proc Natl Acad Sci US A. 2012;109(37):15024-15029.

31. Mannick JB, et al. TORC1 inhibition enhances immune function and reduces infections in the elderly. Sci Transl Med. 2018;10(449):eaaq1564.

32. Qin X, Jiang B, Zhang Y. 4E-BP1, a multifactor regulated multifunctional protein. Cell Cycle. 2016;15(6):781-786.

33. Borden KL. The eukaryotic translation initiation factor eIF4E wears a "cap" for many occasions. Translation (Austin). 2016;4(2):e1220899.

34. Bhat M, Robichaud N, Hulea L, Sonenberg N, Pelletier J, Topisirovic I. Targeting the translation machinery in cancer. Nat Rev Drug Discov. 2015;14(4):261-278.

35. Chu J, Pelletier J. Therapeutic opportunities in eukaryotic translation. Cold Spring Harb Perspect Biol.2018;10(6):a032995.

36. Rousseau D, Gingras AC, Pause A, Sonenberg $\mathrm{N}$. The eIF4E-binding proteins 1 and 2 are negative regulators of cell growth. Oncogene. 1996;13(11):2415-2420.

37. Sonenberg N, Gingras AC. The mRNA 5' cap-binding protein eIF $4 \mathrm{E}$ and control of cell growth. Curr Opin Cell Biol. 1998;10(2):268-275.

38. Colina R, et al. Translational control of the innate immune response through IRF-7. Nature. 2008;452(7185):323-328.

39. Truitt ML, et al. Differential requirements for eIF $4 \mathrm{E}$ dose in normal development and cancer. Cell. 2015;162(1):59-71.

40. Truitt ML, Ruggero D. New frontiers in translational control of the cancer genome. Nat Rev Cancer. 2016;16(5):288-304.

41. Hinnebusch AG, Ivanov IP, Sonenberg N. Transla- tional control by 5 - -untranslated regions of eukaryotic mRNAs. Science. 2016;352(6292):1413-1416.

42. Leppek K, Das R, Barna M. Functional 5' UTR mRNA structures in eukaryotic translation regulation and how to find them. Nat Rev Mol Cell Biol. 2018;19(3):158-174.

43. Assouline S, et al. Molecular targeting of the oncogene eIF4E in acute myeloid leukemia (AML): a proof-of-principle clinical trial with ribavirin. Blood. 2009;114(2):257-260.

44. Thoreen CC, Chantranupong L, Keys HR, Wang T, Gray NS, Sabatini DM. A unifying model for mTORC1-mediated regulation of mRNA translation. Nature. 2012;485(7396):109-113.

45. Koromilas AE, Lazaris-Karatzas A, Sonenberg N. mRNAs containing extensive secondary structure in their $5^{\prime}$ non-coding region translate efficiently in cells overexpressing initiation factor eIF-4E. EMBO J.1992;11(11):4153-4158.

46. González-Terán B, et al. Eukaryotic elongation factor 2 controls TNF- $\alpha$ translation in LPS-induced hepatitis. JClin Invest. 2013;123(1):164-178.

47. Uttam S, et al. Translational profiling of dorsal root ganglia and spinal cord in a mouse model of neuropathic pain. Neurobiol Pain. 2018;4:35-44.

48. Gkogkas CG, et al. Autism-related deficits via dysregulated eIF4E-dependent translational control. Nature. 2013;493(7432):371-377.

49. Galatro TF, et al. Transcriptomic analysis of purified human cortical microglia reveals age-associated changes. Nat Neurosci. 2017;20(8):1162-1171.

50. Manning BD, Cantley LC. Rheb fills a GAP between TSC and TOR. Trends Biochem Sci. 2003;28(11):573-576.

51. Zou J, et al. Rheb1 is required for $\mathrm{mTORC1}$ and myelination in postnatal brain development. Dev Cell.2011;20(1):97-108.

52. Shi J, Hua L, Harmer D, Li P, Ren G. Cre driver mice targeting macrophages. Methods Mol Biol. 2018;1784:263-275

53. Melser S, et al. Rheb regulates mitophagy induced by mitochondrial energetic status. Cell Metab. 2013;17(5):719-730.

54 . Tyagi R, et al. Rheb inhibits protein synthesis by activating the PERK-eIF2 $\alpha$ signaling cascade. Cell Rep. 2015;10(5):684-693.

55. Tamai T, et al. Rheb (Ras homologue enriched in brain)-dependent mammalian target of rapamycin complex 1 (mTORC1) activation becomes indispensable for cardiac hypertrophic growth after early postnatal period. J Biol Chem. 2013;288(14):10176-10187.

56. Thoreen CC, et al. An ATP-competitive mammalian target of rapamycin inhibitor reveals rapamycin-resistant functions of mTORC1.J Biol Chem. 2009;284(12):8023-8032.

57. Gingras AC, et al. Hierarchical phosphorylation of the translation inhibitor 4E-BP1. Genes Dev. 2001;15(21):2852-2864.

58. Gingras AC, et al. Regulation of 4E-BP1 phosphorylation: a novel two-step mechanism. Genes Dev. 1999;13(11):1422-1437.

59. Mothe-Satney I, Yang D, Fadden P, Haystead TA, Lawrence JC. Multiple mechanisms control phosphorylation of PHAS-I in five (S/T)P sites that govern translational repression. Mol Cell Biol. 2000;20(10):3558-3567.

60. Choo AY, Yoon SO, Kim SG, Roux PP, Blenis 
J. Rapamycin differentially inhibits $\mathrm{S6Ks}$ and 4E-BP1 to mediate cell-type-specific repression of mRNA translation. Proc Natl Acad Sci US A. 2008;105(45):17414-17419.

61. Feldman ME, et al. Active-site inhibitors of MTOR target rapamycin-resistant outputs of mTORC1 and mTORC2. PLoS Biol. 2009;7(2):e38.

62. Livingstone M, Bidinosti M. Rapamycin-insensitive mTORC1 activity controls eIF4E:4E-BP1 binding. F1000Res. 2012;1:4.

63. Herbert TP, Tee AR, Proud CG. The extracellular signal-regulated kinase pathway regulates the phosphorylation of 4E-BP1 at multiple sites. JBiol Chem. 2002;277(13):11591-11596.

64. Moerke NJ, et al. Small-molecule inhibition of the interaction between the translation initiation factors eIF4E and eIF4G. Cell. 2007;128(2):257-267.

65. Sekiyama N, Arthanari H, Papadopoulos E, Rodriguez-Mias RA, Wagner G, Léger-Abraham M. Molecular mechanism of the dual activity of 4EGI1: dissociating eIF $4 \mathrm{G}$ from eIF $4 \mathrm{E}$ but stabilizing the binding of unphosphorylated 4E-BP1. Proc Natl Acad Sci U S A. 2015;112(30):E4036-E4045.

66. Pyronnet S, Imataka H, Gingras AC, Fukunaga R, Hunter T, Sonenberg N. Human eukaryotic translation initiation factor $4 \mathrm{G}$ (eIF $4 \mathrm{G}$ ) recruits mnk1 to phosphorylate eIF4E. EMBO J. 1999;18(1):270-279.

67. Waskiewicz AJ, Johnson JC, Penn B, Mahalingam M, Kimball SR, Cooper JA. Phosphorylation of the cap-binding protein eukaryotic translation initiation factor $4 \mathrm{E}$ by protein kinase Mnk1 in vivo. $\mathrm{Mol}$ Cell Biol. 1999;19(3):1871-1880.

68. Scheper GC, Morrice NA, Kleijn M, Proud CG. The mitogen-activated protein kinase signal-integrating kinase Mnk 2 is a eukaryotic initiation factor $4 \mathrm{E}$ kinase with high levels of basal activity in mammalian cells. Mol Cell Biol. 2001;21(3):743-754.

69. Shveygert M, Kaiser C, Bradrick SS, Gromeier M. Regulation of eukaryotic initiation factor $4 \mathrm{E}$ (eIF4E) phosphorylation by mitogen-activated protein kinase occurs through modulation of Mnk1-eIF4G interaction. Mol Cell Biol. 2010;30(21):5160-5167.

70. Kentsis A, Topisirovic I, Culjkovic B, Shao L, Borden KL. Ribavirin suppresses eIF4E-mediated oncogenic transformation by physical mimicry of the 7-methyl guanosine mRNA cap. Proc Natl Acad Sci
US A. 2004;101(52):18105-18110.

71. Olah M, et al. A transcriptomic atlas of aged human microglia. Nat Commun. 2018;9(1):539.

72. Keren-Shaul H, et al. A unique microglia type associated with restricting development of Alzheimer's disease. Cell. 2017;169(7):1276-1290.e17.

73. Mass E, et al. A somatic mutation in erythro-myeloid progenitors causes neurodegenerative disease. Nature. 2017;549(7672):389-393.

74. Venegas C, et al. Microglia-derived ASC specks cross-seed amyloid-beta in Alzheimer's disease. Nature. 2017;552(7685):355-361.

75. Raj DD, et al. Priming of microglia in a DNA-repair deficient model of accelerated aging. Neurobiol Aging. 2014;35(9):2147-2160.

76. Shimobayashi M, Hall MN. Making new contacts: the mTOR network in metabolism and signalling crosstalk. Nat Rev Mol Cell Biol. 2014;15(3):155-162.

77. Junnila RK, List EO, Berryman DE, Murrey JW, Kopchick JJ. The GH/IGF-1 axis in ageing and longevity. Nat Rev Endocrinol. 2013;9(6):366-376.

78. Katoh-Semba R, Semba R, Takeuchi IK, Kato K. Age-related changes in levels of brain-derived neurotrophic factor in selected brain regions of rats, normal mice and senescence-accelerated mice: a comparison to those of nerve growth factor and neurotrophin-3. Neurosci Res. 1998;31(3):227-234.

79. Sonenberg N, Hinnebusch AG. Regulation of translation initiation in eukaryotes: mechanisms and biological targets. Cell. 2009;136(4):731-745.

80. Altmann M, Edery I, Sonenberg N, Trachsel H. Purification and characterization of protein synthesis initiation factor eIF-4E from the yeast Saccharomyces cerevisiae. Biochemistry. 1985;24(22):6085-6089.

81. Herdy B, et al. Translational control of the activation of transcription factor NF- $\mathrm{kB}$ and production of type I interferon by phosphorylation of the translation factor eIF4E. Nat Immunol. 2012;13(6):543-550.

82. Hsieh AC, et al. The translational landscape of mTOR signalling steers cancer initiation and metastasis. Nature. 2012;485(7396):55-61.

83. Elia A, Constantinou C, Clemens MJ. Effects of protein phosphorylation on ubiquitination and stability of the translational inhibitor protein 4E-BP1. Oncogene. 2008;27(6):811-822.

84. Papadopoli D, et al. mTOR as a central regulator of lifespan and aging. F100ORes. 2019;8:F1000
Faculty Rev-998.

85. Johnson SC, Rabinovitch PS, Kaeberlein M. mTOR is a key modulator of ageing and age-related disease. Nature. 2013;493(7432):338-345.

86. Rangaraju S, Verrier JD, Madorsky I, Nicks J, Dunn WA, Notterpek L. Rapamycin activates autophagy and improves myelination in explant cultures from neuropathic mice. J Neurosci. 2010;30(34):11388-11397.

87. Thomson AW, Turnquist HR, Raimondi G. Immunoregulatory functions of $\mathrm{mTOR}$ inhibition. Nat RevImmunol. 2009;9(5):324-337.

88. Weichhart T, Hengstschläger M, Linke M. Regulation of innate immune cell function by mTOR. Nat RevImmunol. 2015;15(10):599-614.

89. Mannick JB, et al. mTOR inhibition improves immune function in the elderly. Sci Transl Med. 2014;6(268):268ra179.

90. Peng H, et al. Distinct roles of Rheb and Raptor in activating mTOR complex 1 for the self-renewal of hematopoietic stem cells. Biochem Biophys Res Commun. 2018;495(1):1129-1135.

91. Andrews S. AndrewsFastQC: a quality control tool for high throughput sequence data. Babraham Institute. 2010. http://www.bioinformatics.babraham. ac.uk/projects/fastqc. Accessed October 19, 2020.

92. Harrow J, et al. GENCODE: the reference human genome annotation for The ENCODE Project. Genome Res. 2012;22(9):1760-1774.

93. Frankish A, et al. GENCODE reference annotation for the human and mouse genomes. Nucleic Acids Res. 2019;47(D1):D766-D773.

94. Dobin A, et al. STAR: ultrafast universal RNA-seq aligner. Bioinformatics. 2013;29(1):15-21.

95. Lawrence $\mathrm{M}$, et al. Software for computing and annotating genomic ranges. PLoS Comput Biol. 2013;9(8):e1003118.

96. Love MI, Huber W, Anders S. Moderated estimation of fold change and dispersion for RNA-seq data with DESeq2. Genome Biol. 2014;15(12):550.

97. Benjamini Y. Controlling the false discovery rate: a practical and powerful approach to multiple testing. JR Stat Soc Series B Stat Methodol. 1995;57(1):289-300.

98. Abdi H. The Bonferonni and Šidák corrections for multiple comparisons. In: Salkind N. Encyclopedia of Measurement and Statistics. Sage; 2007:103-107. 